\title{
Binary-black-hole initial data with nearly extremal spins
}

\author{
Geoffrey Lovelace, ${ }^{1,2}$ Robert Owen, ${ }^{1,2}$ Harald P. Pfeiffer, ${ }^{2}$ and Tony $\mathrm{Chu}^{2}$ \\ ${ }^{1}$ Center for Radiophysics and Space Research, Cornell University, Ithaca, New York 14853, USA \\ ${ }^{2}$ Theoretical Astrophysics 130-33, California Institute of Technology, Pasadena, California 91125, USA
}

(Received 27 May 2008; published 10 October 2008)

There is a significant possibility that astrophysical black holes with nearly extremal spins exist. Numerical simulations of such systems require suitable initial data. In this paper, we examine three methods of constructing binary-black-hole initial data, focusing on their ability to generate black holes with nearly extremal spins: (i) Bowen-York initial data, including standard puncture data (based on conformal flatness and Bowen-York extrinsic curvature), (ii) standard quasiequilibrium initial data (based on the extended-conformal-thin-sandwich equations, conformal flatness, and maximal slicing), and (iii) quasiequilibrium data based on the superposition of Kerr-Schild metrics. We find that the two conformally flat methods (i) and (ii) perform similarly, with spins up to about 0.99 obtainable at the initial time. However, in an evolution, we expect the spin to quickly relax to a significantly smaller value around 0.93 as the initial geometry relaxes. For quasiequilibrium superposed Kerr-Schild data [method (iii)], we construct initial data with initial spins as large as 0.9997. We evolve superposed Kerr-Schild data sets with spins of 0.93 and 0.97 and find that the spin drops by only a few parts in $10^{4}$ during the initial relaxation; therefore, we expect that superposed Kerr-Schild initial data will allow evolutions of binary black holes with relaxed spins above 0.99 . Along the way to these conclusions, we also present several secondary results: the power-law coefficients with which the spin of puncture initial data approaches its maximal possible value; approximate analytic solutions for large spin puncture data; embedding diagrams for single spinning black holes in methods (i) and (ii); nonunique solutions for method (ii). All of the initial-data sets that we construct contain subextremal black holes, and when we are able to push the spin of the excision boundary surface into the superextremal regime, the excision surface is always enclosed by a second, subextremal apparent horizon. The quasilocal spin is measured by using approximate rotational Killing vectors, and the spin is also inferred from the extrema of the intrinsic scalar curvature of the apparent horizon. Both approaches are found to give consistent results, with the approximate-Killing-vector spin showing the least variation during the initial relaxation.

DOI: 10.1103/PhysRevD.78.084017

PACS numbers: 04.25.D-, 02.70.Hm, 04.20.Ex, 04.25.dg

\section{INTRODUCTION}

There is a significant possibility that black holes with nearly extremal spins exist; by "nearly extremal," we mean that the spin $S$ and mass $M$ of the hole satisfy $0.95 \lesssim$ $S / M^{2} \lesssim 1$. Some models of black-hole accretion [1-3] predict that most black holes will have nearly extremal spins, and observational evidence for black holes with nearly extremal spins includes, e.g., estimates of blackhole spins in quasars [4] and estimates of the spin of a black hole in a certain binary x-ray source [5]. There is considerable uncertainty about whether black holes do in fact typically have nearly extremal spins; e.g., some models [68] of black-hole accretion do not lead to large spins. This uncertainty could be reduced by measuring the holes' spins directly using gravitational waves.

This prospect of detecting the gravitational waves emitted by colliding black holes, possibly with nearly extremal spins, motivates the goal of simulating these spacetimes numerically. Indeed, one focus of intense research has been spinning black-hole binaries, including the discovery of dramatic kicks when two spinning black holes merge [917] as well as some initial exploration of the orbital dy- namics of spinning binaries [18-23]. All of these simulations start from puncture initial data as introduced by Brandt and Brügmann [24].

The simplifying assumptions employed in puncture initial data make it impossible to construct black holes with spins arbitrarily close to unity. The numerical value of the fastest obtainable spin depends on which dimensionless ratio is chosen to characterize "black-hole spin." Often, dimensionless spin is defined based on quasilocal properties of the black hole

$$
\chi:=\frac{S}{M^{2}},
$$

where $S$ is taken to be nonnegative and is a suitable quasilocal spin (e.g., obtained using approximate rotational Killing vectors on the apparent horizon as described, for example, in Appendix A) and $M$ is a suitable quasilocal mass. The latter may be obtained from Christodoulou's formula relating spin, area and mass of a Kerr black hole

$$
M^{2}:=M_{\mathrm{irr}}^{2}+\frac{S^{2}}{4 M_{\mathrm{irr}}^{2}}
$$


where we define the irreducible mass in terms of the area $A$ of the apparent horizon by $M_{\text {irr }}:=\sqrt{A / 16 \pi}$.

The quantity $\chi$ is not preserved during an evolution. Specifically, most black-hole initial data are not exactly in equilibrium, which leads to transients and emission of an artificial pulse of gravitational radiation early in numerical simulations. The geometry in the vicinity of the black holes relaxes on a time scale $t_{\text {relax }}$ (typically a few $M$ ), and during this relaxation, the spin changes by

$$
\Delta \chi:=\chi(t=0)-\chi\left(t_{\text {relax }}\right) .
$$

When constructing a single spinning black hole with standard puncture data [24], for instance, $\chi(t=0) \lesssim 0.98$, which seems encouragingly large. However Dain et al. $[25,26]$ evolved standard puncture data with initial spin close to this limit, and they find that the spin rapidly drops to $\chi\left(t_{\text {relax }}\right) \approx 0.93$, i.e., $\Delta \chi \approx 0.05$.

For single-black-hole spacetimes, another widely used dimensionless spin measure is the ratio of total angular momentum ${ }^{1} J_{\mathrm{ADM}}$ and Arnowitt-Deser-Misner (ADM) energy $E_{\mathrm{ADM}}$ :

$$
\varepsilon_{J}:=\frac{J_{\mathrm{ADM}}}{E_{\mathrm{ADM}}^{2}} .
$$

Dain et al. noted that $\chi\left(t_{\text {relax }}\right)$ is close to $\varepsilon_{J}$ and explained this result as follows: The spacetime is axisymmetric, which implies that the angular momentum $J_{\mathrm{ADM}}$ is conserved and that the black hole's spin equals $J_{\mathrm{ADM}}$. Moreover, so long as a negligible fraction of the spacetime's energy is carried off by the spurious radiation, the hole's quasilocal mass will relax to a value of $E_{\mathrm{ADM}}$, giving $\chi\left(t_{\text {relax }}\right) \approx \varepsilon_{J}$. Thus conformally flat Bowen-York data cannot be used to simulate black holes with nearly extremal equilibrium spins, even though the initial spins can be made fairly close to $\chi=1$.

This paper examines three different approaches of constructing black-hole initial data with nearly extremal spin. First, we revisit puncture initial data and inversionsymmetric Bowen-York initial data. We show that for a single, spinning black hole at rest, both approaches are identical, and we determine spin limits based purely on initial data more accurately than before:

$$
\varepsilon_{J} \leq 0.928200, \quad \chi(t=0) \leq 0.9837 .
$$

We show that the limiting values of $\varepsilon_{J}$ and $\chi(t=0)$ are approached as power laws of the spin parameter (curiously, with different powers). We furthermore give insight into the geometric structure of these high-spin Bowen-York initial-data sets through numerical study and approximate

\footnotetext{
${ }^{1}$ We define here $J_{\mathrm{ADM}}$ by an ADM-like surface integral at infinity; in axisymmetry this definition coincides with the standard Komar integral for angular momentum (see Sec. II B for details.)
}

analytical solutions and find that a cylindrical throat forms which lengthens logarithmically with the spin parameter.

Second, we investigate the high-spin limit of another popular approach of constructing initial data, the quasiequilibrium formalism [27-31] based on the conformal thin sandwich equations [32,33]. For the standard choices of conformal flatness and maximal slicing, we are able to construct initial data with spins somewhat larger than the standard Bowen-York limits given in Eq. (5):

$$
\varepsilon_{J} \lesssim 0.94, \quad \chi(t=0) \lesssim 0.99 .
$$

Once again $\varepsilon_{J}$ is much lower than $\chi(t=0)$, which suggests that these data sets lead to equilibrium spins of approximate magnitude $\chi \approx 0.94$. Interestingly, these families of initial data are found to exhibit nonunique solutions [34-36], and the largest spins are obtained along the upper branch.

The third approach also utilizes the quasiequilibrium formalism [27-31], but this time we make use of the freedom to choose arbitrary background data. Specifically, we choose background data as a superposition of two KerrSchild metrics. This approach is based on the original proposal of Matzner and collaborators [37,38] and was first carried over into the conformal thin sandwich equations in Ref. [39]; also, background data consisting of a single, nonspinning Kerr-Schild black hole was used to construct initial data for a black-hole-neutron-star binary in Ref. [40]. For single black holes, these data simply reduce to the analytical Kerr solution. For binary black holes, we construct initial data with spins as large as

$$
\chi(t=0)=0.9997 .
$$

We also present evolutions, demonstrating that our rapidly spinning initial-data sets remain rapidly spinning after the numerical evolution relaxes. In particular, we evolve an orbiting binary with $\chi(t=0)=0.9275$ and a head-on merger with $\chi(t=0)=0.9701$. In both cases, $\mid \Delta \chi / \chi(t=$ $0) \mid$ is significantly smaller than $10^{-3}$. We conclude that the conformally curved superposed Kerr-Schild (SKS) initial data we present in this paper, in contrast with conformally flat Bowen-York data, are suitable for simulating binary black holes with nearly extremal spins.

Throughout the paper, we use two different techniques to measure the dimensionless spin of black holes, which are described in the appendixes. The first (Appendix A) technique uses the standard surface-integral based on an approximate rotational Killing vector of the apparent horizon. We compute the approximate Killing vector with a variation of the technique introduced by Cook and Whiting [41], extended with new normalization conditions of the approximate Killing vector, and we denote the resulting spin "AKV spin" $\chi_{\mathrm{AKV}}$. The second approach (Appendix B) is based on the shape of the horizon in the form of its scalar curvature; specifically, the spin magnitudes are inferred from the minimum and maximum of the 
intrinsic Ricci scalar curvature of the horizon. We call the spin inferred in this way the "scalar-curvature spin," and we label the spin magnitudes inferred from the scalarcurvature minimum and maximum as $\chi_{\mathrm{SC}}^{\min }$ and $\chi_{\mathrm{SC}}^{\max }$, respectively. Typically, binary-black-hole initial data produce holes that are initially not in equilibrium. Therefore, we use only the AKV spin to measure the initial black-hole spin (Secs. III and IV.) We use both the AKV and the scalarcurvature spin when we measure the spin after the holes have relaxed to equilibrium (Sec. V).

We also monitor whether any of the constructed initialdata sets have superextremal spins, as this may shed light, for example, on the cosmic censorship conjecture. When using the Christodoulou formula [Eq. (2)] to define $M$, the quasilocal dimensionless spin $\chi$ is by definition bounded [42], $\chi \leq 1$. This can be seen most easily by introducing the parameter $\zeta$, defined as

$$
\zeta:=\frac{S}{2 M_{\mathrm{irr}}^{2}}
$$

and then rewriting $\chi$ as

$$
\chi=1-\frac{(1-\zeta)^{2}}{1+\zeta^{2}}
$$

The ratio $\chi$ is therefore not useful to diagnose superextremal black holes. A more suitable diagnostic is found in the parameter $\zeta$. For Kerr black holes, the first term on the right-hand side of Eq. (2) is always smaller or equal to the second, with equality only for extremal spin; i.e., $\zeta \leq$ 1 , with equality for extremal spin. This motivates an alternative definition of extremality [42]: A black hole is said to be superextremal if the second term in Eq. (2) is larger than the first one, i.e., if $\zeta>1$. In this paper, we monitor $\zeta$, which we call the spin-extremality parameter, along with the dimensionless spin $\chi$. We find instances where $\zeta$ exceeds unity. Before this happens, however, a larger, subextremal $(\zeta<1)$ apparent horizon appears, enclosing the smaller, superextremal horizon (Sec. IV B, Fig. 12).

This paper is organized as follows. Section II summarizes the various formalisms that we use to construct initial data. Section III investigates single-black-hole initial data, followed by the construction of binary-black-hole initial data in Sec. IV. Section V presents binary-black-hole evolutions that show the good properties of superposed KerrSchild data and the various spin diagnostics. We summarize and discuss our results in Sec. VI. Finally, Appendixes A and B present our techniques to define black-hole spin.

\section{INITIAL-DATA FORMALISM}

Before constructing initial data for rapidly spinning single (Sec. III) and binary (Sec. IV) black holes, we first summarize the initial-data formalisms we will use. After laying some general groundwork in Sec. II A, we describe Bowen-York initial data (including puncture initial data) in
Sec. II B and quasiequilibrium extended-conformal-thinsandwich data in Sec. II C.

\section{A. Extrinsic curvature decomposition}

Initial-data sets for Einstein's equations are given on a spatial hypersurface $\Sigma$ and must satisfy the constraint equations

$$
\begin{gathered}
R+K^{2}-K_{i j} K^{i j}=0, \\
\nabla_{j}\left(K^{i j}-g^{i j} K\right)=0 .
\end{gathered}
$$

Here, $g_{i j}$ is the induced metric of the slice $\Sigma$, with covariant derivative $\nabla_{i}, R:=g^{i j} R_{i j}$ denotes the trace of the Ricci tensor $R_{i j}$, and $K_{i j}$ denotes the extrinsic curvature of the slice $\Sigma$ as embedded into the spacetime manifold $\mathcal{M}$.

The constraint equations (10) and (11) can be transformed into elliptic partial differential equations using a conformal transformation, e.g., [33]. One introduces a conformal metric $\tilde{g}_{i j}$ via

$$
g_{i j}=\psi^{4} \tilde{g}_{i j},
$$

with the strictly positive conformal factor $\psi>0$. Substituting Eq. (12) into Eq. (10) yields an elliptic equation for $\psi$. One furthermore decomposes the extrinsic curvature into trace and trace-free parts

$$
K^{i j}=A^{i j}+\frac{1}{3} g^{i j} K
$$

and splits off a longitudinal part from the trace-free extrinsic curvature

$$
A^{i j}=\frac{1}{\sigma}(\llbracket V)^{i j}+M^{i j} .
$$

In Eq. (14), $\sigma$ is a strictly positive weight function, the longitudinal operator is defined as $(\mathbb{L} V)^{i j}=2 \nabla^{(i} V^{j)}-$ $\frac{2}{3} g^{i j} \nabla_{k} V^{k}$, and $M^{i j}$ is symmetric and trace-free. ${ }^{2}$ Finally, one introduces the conformally scaled quantities $\sigma=\psi^{6} \tilde{\sigma}$ and $M^{i j}=\psi^{-10} \tilde{M}^{i j}$, which allows the momentum constraint [Eq. (11)] to be rewritten completely in terms of conformal quantities:

$$
\begin{gathered}
A^{i j}=\psi^{-10} \tilde{A}^{i j}, \\
\tilde{A}^{i j}=\frac{1}{\tilde{\sigma}}(\tilde{\mathbb{L}} V)^{i j}+\tilde{M}^{i j} .
\end{gathered}
$$

The Hamiltonian and momentum constraints then become

$$
\begin{gathered}
\tilde{\nabla}^{2} \psi-\frac{1}{8} \tilde{R}-\frac{1}{12} K^{2} \psi^{5}+\frac{1}{8} \tilde{A}_{i j} \tilde{A}^{i j} \psi^{-7}=0, \\
\tilde{\nabla}_{j}\left(\frac{1}{\tilde{\sigma}}(\tilde{\mathbb{L}} V)^{i j}\right)-\frac{2}{3} \psi^{6} \tilde{\nabla}^{i} K+\tilde{\nabla}_{j} \tilde{M}^{i j}=0 .
\end{gathered}
$$

\footnotetext{
${ }^{2}$ It is also possible, but not necessary, to require that $M^{i j}$ is divergence-free.
} 
Given choices for $\tilde{M}^{i j}, K, \tilde{g}_{i j}$ and $\tilde{\sigma}$, and also boundary conditions, one can solve Eqs. (17) and (18) for $\psi$ and $V^{i}$ and then assemble the (constraint-satisfying) initial data $g_{i j}$ and $K^{i j}$.

Many important approaches to construct binary-blackhole initial data can be cast in this form. The various approaches differ in the choices for the freely specifiable parts and the boundary conditions. Some choices of free data aim for simplicity, such as Bowen-York initial data. Other approaches aim to preserve freedom, resulting in more complicated sets of equations but also more flexibility to control properties of the resulting initial data. The quasiequilibrium extended-conformal-thin-sandwich approach falls into this second category, and we will exploit precisely its inherent freedom in choosing the free data to construct black holes with nearly extremal spins.

\section{B. Bowen-York initial data}

In this section, we describe two approaches of constructing initial data based on the well-known Bowen-York extrinsic curvature. These two approaches, puncture data and inversion-symmetric data, differ in how they treat the coordinate singularity at $r=0$; both can be obtained from the general procedure outlined in Sec. II A by setting $\tilde{\sigma} \equiv$ $1, K \equiv 0, \tilde{M}^{i j} \equiv 0$ and by using a conformally flat metric

$$
\tilde{g}_{i j}=f_{i j} \text {. }
$$

The momentum constraint [Eq. (18)] then reduces to $\tilde{\nabla}_{j}(\tilde{\mathbb{L}} V)^{i j}=0$, which is solved by choosing the analytical Bowen-York solutions [43,44].

The Bowen-York solutions can be written down most conveniently in Cartesian coordinates, $f_{i j}=\delta_{i j}$ :

$$
\begin{gathered}
V_{P}^{i}=-\frac{1}{4 r}\left[7 P^{i}+n^{i} P^{k} n_{k}\right], \\
V_{S}^{i}=-\frac{1}{r^{2}} \epsilon_{l m}^{i} S^{l} n^{m},
\end{gathered}
$$

where $r=\left(x^{i} x^{j} \delta_{i j}\right)^{1 / 2}$ is the coordinate distance to the origin and $n^{i}=x^{i} / r$ is the coordinate unit vector pointing from the origin to the point under consideration. The spatially constant vectors $P^{i}$ and $S^{i}$ parametrize the solutions $^{3}$

$$
\begin{gathered}
\tilde{A}_{P}^{i j}=\frac{3}{2 r^{2}}\left[2 P^{(i} n^{j)}-\left(\delta^{i j}-n^{i} n^{j}\right) P_{k} n^{k}\right] \\
\tilde{A}_{S}^{i j}=\frac{6}{r^{3}} n_{(i} \epsilon_{j) k l} S^{k} n^{l}
\end{gathered}
$$

\footnotetext{
${ }^{3}$ In Cartesian coordinates, upper and lower indices are equivalent, so index positioning in Eqs. (20)-(23) is unimportant. To find $\tilde{A}_{P / S}^{i j}$ in another coordinate system, first compute the Cartesian components Eqs. (20)-(23) and then apply the desired coordinate transformation.
}

The conformal factor $\psi$ is then determined by the Hamiltonian constraint [Eq. (17)], which simplifies to

$$
\tilde{\nabla}^{2} \psi+\frac{1}{8} \psi^{-7} \tilde{A}^{i j} \tilde{A}_{i j}=0 .
$$

We would like to recover an asymptotically flat space; this implies the boundary condition $\psi \rightarrow 1$ as $r \rightarrow \infty$.

This boundary condition makes it possible to evaluate the linear ADM-momentum and ADM-like angular momentum of Bowen-York initial data without solving Eq. (24). These quantities are defined by surface integrals at infinity

$$
J_{(\xi)}=\frac{1}{8 \pi} \oint_{\infty}\left(K_{i j}-g_{i j} K\right) \xi^{i} s^{j} d A
$$

where $s^{i}$ is the outward-pointing unit normal to the integration sphere. ${ }^{4}$ By letting $\psi \rightarrow 1$ in Eq. (15), one can replace $K_{i j}$ by $\tilde{A}_{i j}$ and then evaluate the resulting integrals. The choice of vector $\xi^{i}$ determines which quantity is computed: For instance, $\xi=\hat{e}_{x}$ corresponds to the $x$ component of the linear ADM momentum; $\xi=\partial_{\phi}=-x \hat{e}_{y}+$ $y \hat{e}_{x}$ yields the $z$ component of the ADM-like angular momentum. ${ }^{5}$ For Eqs. (22) and (23), the results are $P_{\mathrm{ADM}}^{i}=P^{i}$ and $J_{\mathrm{ADM}}^{i}=S^{i}$, respectively.

The ADM energy is given by the expression

$$
E_{\mathrm{ADM}}=\frac{1}{16 \pi} \oint_{\infty} \nabla_{j}\left(G_{i}{ }^{j}-\delta_{i}{ }^{j} G\right) s^{i} d A,
$$

where $G_{i j}:=g_{i j}-f_{i j}, G:=G_{i j} g^{i j}$. For conformal flatness, Eq. (26) reduces to

$$
E_{\mathrm{ADM}}=-\frac{1}{2 \pi} \oint_{\infty} \partial_{r} \psi d A .
$$

The derivative of the conformal factor is known only after Eq. (24) is solved; therefore, in contrast with the linear and angular momenta, $E_{\mathrm{ADM}}$ can be computed only after solving the Hamiltonian constraint.

We now turn our attention to inner boundary conditions. $\tilde{A}_{P}^{i j}$ and $\tilde{A}_{S}^{i j}$ are singular at $r=0$. This singularity is interpreted as a second asymptotically flat universe; when solving Eq. (24), this can be incorporated in two ways:

\footnotetext{
${ }^{4}$ At infinity, the normal to the sphere $s^{i}$ is identical to the coordinate radial unit vector $n^{i}$.

${ }^{5} \mathrm{As}$ is common in the numerical relativity community, we introduce the phrase "ADM angular momentum" to refer to an angular momentum defined at spatial infinity in the manner of the other conserved ADM quantities of asymptotically flat spacetimes [45], despite the fact that (at least to our knowledge), no such quantity is widely agreed to rigorously exist in general, due to the supertranslation ambiguity that exists in four spacetime dimensions. For recent research on this issue see [46] and references therein. In the present paper, this subtlety can be ignored, because we only compute this quantity in truly axisymmetric spacetimes, with $\vec{\xi}$ the global axisymmetry generator, so that $J_{\mathrm{ADM}}$ coincides with the standard Komar integral for angular momentum.
} 
(i) Inversion symmetry.-The demand that the solution be symmetric under inversion at a sphere with radius $R_{\text {inv }}$ centered on the origin [44] results in a boundary condition for $\psi$ at $r=R_{\text {inv }}$, namely, $\partial \psi / \partial r=$ $-\psi /\left(2 R_{\text {inv }}\right)$. The Hamiltonian constraint Eq. (24) is solved only in the exterior of the sphere $r \geq$ $R_{\text {inv }}$, and the solution in the interior can be recovered from inversion symmetry [44], e.g.,

$$
\psi\left(x^{i}\right)=\frac{R_{\mathrm{inv}}}{r} \psi\left(\frac{R_{\mathrm{inv}}^{2}}{r^{2}} x^{i}\right) .
$$

(ii) Puncture data.-One demands [24] the appropriate singular behavior of $\psi$ for $r \rightarrow 0$ to ensure that the second asymptotically flat end is indeed flat. That is, $\psi$ must behave as

$$
\psi\left(x^{i}\right)=\frac{m_{p}}{2 r}+1+u\left(x^{i}\right)
$$

for some positive parameter $m_{p}$ (the "puncture mass") and function $u\left(x^{i}\right)$ that is finite and continuous in $\mathbb{R}^{3}$ and approaches 0 as $r \rightarrow \infty$. Equation (24) then implies an equation for $u$ that is finite everywhere and can be solved without any inner boundaries:

$$
\tilde{\nabla}^{2} u=-\frac{1}{8} \frac{\tilde{A}_{i j} \tilde{A}^{i j} r^{7}}{\left(r+\frac{m_{p}}{2}+u r\right)^{7}} .
$$

The majority of binary black hole simulations use puncture data; see, e.g., Refs. [9-23].

Both approaches allow specification of multiple black holes at different locations, each with different spin and momentum parameters $S^{i}$ and $P^{i}$. For puncture data this is almost trivial; this accounts for the popularity of puncture data as initial data for black-hole simulations. In contrast, for inversion-symmetric data, one needs to employ a rather cumbersome imaging procedure ${ }^{6}$ (see, e.g., [47] for details).

For a single spinning black hole at the origin, the extrinsic curvature $\tilde{A}_{S}^{i j}$ given by Eq. (23) is identical for inversion-symmetric and puncture data. For inversionsymmetric data, the conformal factor has the usual falloff at large radii:

$$
\psi\left(x^{i}\right)=1+\frac{E_{\mathrm{ADM}}}{2 r}+\mathcal{O}\left(r^{-2}\right), \quad \text { as } r \rightarrow \infty .
$$

Using Eq. (28) we find the behavior of $\psi$ as $r \rightarrow 0$ :

$$
\psi\left(x^{i}\right)=\frac{R_{\mathrm{inv}}}{r}+\frac{E_{\mathrm{ADM}}}{2 R_{\mathrm{inv}}}+\mathcal{O}(r), \quad \text { as } r \rightarrow 0 .
$$

Comparison with Eq. (29) shows that this is precisely the

\footnotetext{
${ }^{6}$ Even for a single black hole with $P^{k} \neq 0$, Eq. (22) has to be augmented by additional terms of $\mathcal{O}\left(r^{-4}\right)$ to preserve inversion symmetry [44].
}

desired behavior for puncture data, if one identifies $R_{\mathrm{inv}}=$ $m_{p} / 2$ and $E /\left(2 R_{\text {inv }}\right)=1+u(0)$. Because puncture data have a unique solution, it follows that for single spinning black holes, puncture data and inversion-symmetric data are identical, provided $m_{p}=2 R_{\text {inv }}$.

For inversion-symmetric initial data for a single, spinning black hole, it is well known [48] that the apparent horizon coincides with the inversion sphere: $r_{\mathrm{AH}}=R_{\mathrm{inv}}$. Therefore, we conclude that for puncture data for a single, spinning black hole, the apparent horizon is an exact coordinate sphere with radius $r_{\mathrm{AH}}=m_{p} / 2$, despite $\tilde{A}_{S}^{i j}$ and $u\left(x^{i}\right)$ not being spherically symmetric.

\section{Quasiequilibrium extended-conformal-thin- sandwich initial data}

Another popular approach of constructing binary-blackhole initial data is the quasiequilibrium extended-conformal-thin-sandwich (QE-XCTS) formalism [27-31]. Instead of emphasizing the extrinsic curvature, the conformal-thin-sandwich formalism [32] emphasizes the spatial metric $g_{i j}$ and its time derivative. Nevertheless, it is equivalent [33] to the extrinsic curvature decomposition outlined in Sec. II A. The vector $V^{i}$ is identified with the shift $\beta^{i}$,

$$
V^{i} \equiv \beta^{i},
$$

and the weight functions $\sigma$ and $\tilde{\sigma}$ are identified (up to factor 2) with the lapse and the conformal lapse, respectively,

$$
\sigma \equiv 2 \alpha, \quad \tilde{\sigma} \equiv 2 \tilde{\alpha} .
$$

The tensor $\tilde{M}_{i j}$ is related to the time derivative of the spatial metric $\tilde{u}_{i j}:=\partial_{t} \tilde{g}_{i j}$ by

$$
\tilde{M}_{i j} \equiv \frac{1}{2 \tilde{\alpha}} \tilde{u}_{i j}
$$

Because $M_{i j}$ is trace-free [Eqs. (13), (15), and (16)], we require $\tilde{u}_{i j}$ to be trace-free.

The conformal-thin-sandwich equations allow control of certain time derivatives in the subsequent evolution of the constructed initial data. If the lapse $\alpha$ and shift $\beta^{i}$ from the initial data are used in the evolution, for instance, then the trace-free part of $\partial_{t} g_{i j}$ will be proportional to $\tilde{u}_{i j}$. Therefore (see Refs. [27,30])

$$
\tilde{u}_{i j} \equiv 0
$$

is a preferred choice for initial-data sets that begin nearly in equilibrium, such as binary-black-hole quasicircular orbits.

The evolution equation for $K$ can be used to derive an elliptic equation for the conformal lapse $\tilde{\alpha}$ (or, equivalently, for $\alpha \psi$ ). Upon specification of

$$
\partial_{t} K \equiv 0,
$$


this fifth elliptic equation is to be solved for $\tilde{\alpha}$ simultaneously with Eqs. (17) and (18); cf. [27,30].

Our numerical code uses the conformal factor $\psi$, the shift $\beta^{i}$, and the product of lapse and conformal factor $\alpha \psi=\tilde{\alpha} \psi^{7}$ as independent variables, in order to simplify the equation for $\partial_{t} K$. Thus, the actual equations being solved take the form

$$
\begin{aligned}
0= & \tilde{\nabla}^{2} \psi-\frac{1}{8} \tilde{R} \psi-\frac{1}{12} K^{2} \psi^{5}+\frac{1}{8} \psi^{-7} \tilde{A}^{i j} \tilde{A}_{i j}, \\
0= & \tilde{\nabla}_{j}\left(\frac{\psi^{7}}{2(\alpha \psi)}(\tilde{\mathbb{L}} \beta)^{i j}\right)-\frac{2}{3} \psi^{6} \tilde{\nabla}^{i} K-\tilde{\nabla}_{j}\left(\frac{\psi^{7}}{2(\alpha \psi)} \tilde{u}^{i j}\right), \\
0= & \tilde{\nabla}^{2}(\alpha \psi)-(\alpha \psi)\left[\frac{\tilde{R}}{8}+\frac{5}{12} K^{4} \psi^{4}+\frac{7}{8} \psi^{-8} \tilde{A}^{i j} \tilde{A}_{i j}\right] \\
& +\psi^{5}\left(\partial_{t} K-\beta^{k} \partial_{k} K\right),
\end{aligned}
$$

with

$$
\tilde{A}_{i j}=\frac{\psi^{7}}{2 \alpha \psi}\left((\tilde{\mathbb{L}} \beta)_{i j}-\tilde{u}_{i j}\right) .
$$

These equations can be solved only after

(1) specifying the remaining free data: i.e., the conformal metric $\tilde{g}_{i j}$ and the trace of the extrinsic curvature $K$ (we chose already $\tilde{u}_{i j} \equiv 0$ and $\partial_{t} K \equiv 0$ ),

(2) choosing an inner boundary $\mathcal{S}$ which excises the black holes' singularities, and also an outer boundary $\mathcal{B}$, and

(3) choosing boundary conditions for $\psi, \alpha \psi$, and $\beta^{i}$ on $\mathcal{B}$ and $\mathcal{S}$.

The initial data are required to be asymptotically flat, and the outer boundary $\mathcal{B}$ is placed at infinity. ${ }^{7}$ If $\tilde{g}_{i j}$ is asymptotically flat, the outer boundary conditions are then

$$
\begin{aligned}
\psi & =1 \quad \text { on } \mathcal{B}, \\
\alpha \psi & =1 \quad \text { on } \mathcal{B}, \\
\beta^{i} & =\left(\Omega_{\mathbf{0}} \times \mathbf{r}\right)^{i}+\dot{a}_{0} r^{i} \quad \text { on } \mathcal{B} .
\end{aligned}
$$

Here $r^{i}$ is the coordinate position vector. The shift boundary condition consists of a rotation (parametrized by the orbital angular velocity $\boldsymbol{\Omega}_{\mathbf{0}}$ ) and an expansion (parametrized by $\dot{a}_{0}$ ); the initial radial velocity is necessary for reducing orbital eccentricity in binary-black-hole initial data [49].

The inner boundary condition on the conformal factor $\psi$ ensures that the excision surfaces $\mathcal{S}$ are apparent horizons [27]:

$$
\begin{aligned}
\tilde{s}^{k} \partial_{k} \psi= & -\frac{\psi^{-3}}{8 \tilde{\alpha}} \tilde{s}^{i} \tilde{s}^{j}\left[(\tilde{\mathbb{L}} \beta)_{i j}-\tilde{u}_{i j}\right]-\frac{\psi}{4} \tilde{h}^{i j} \tilde{\nabla}_{i} \tilde{s}_{j} \\
& +\frac{1}{6} K \psi^{3} \quad \text { on } \mathcal{S} .
\end{aligned}
$$

\footnotetext{
${ }^{7}$ In practice, $\mathcal{B}$ is a sphere with radius $\gtrsim 10^{9}$ times the coordinate radius of the black-hole horizons.
}

Here $\tilde{s}^{i}:=\psi^{2} s^{i}, s^{i}$ is the unit vector normal to $\mathcal{S}$ and $\tilde{h}_{i j}:=\tilde{g}_{i j}-\tilde{s}_{i} \tilde{s}_{j}$ is the induced conformal 2-metric on $\mathcal{S}$.

The inner boundary condition on the shift is

$$
\beta^{i}=\alpha s^{i}-\Omega_{r} \xi^{i} \quad \text { on } \mathcal{S},
$$

where $\xi^{i} s_{i}=0$. The first term on the right-hand side ensures that the apparent horizons are initially at rest; the tangential term determines the black hole's spin [27-29].

References [27-29] chose the sign of the last term in Eq. (40) such that positive values of $\Omega_{r}$ counteract the spin of the corotating holes that are obtained with $\Omega_{r}=0$. Here, we are interested in large spins, and we reverse the sign of the last term in Eq. (40) so that positive, increasing $\Omega_{r}$ results in increasing spins.

Two sets of choices for $\tilde{g}_{i j}, K, \mathcal{S}$, and the boundary condition for $\alpha \psi$ on $\mathcal{S}$ are discussed in the next subsections. Each set of choices will be used to construct binaryblack-hole initial data in Sec. IV.

\section{Conformal flatness and maximal slicing (CFMS)}

The simplest choice for $\tilde{g}_{i j}$ is a flat metric:

$$
\tilde{g}_{i j} \equiv f_{i j} \text {. }
$$

This choice has been used almost exclusively in the previous formulations of binary-black-hole initial data.

The simplest choice for $K$, also commonly used in prior formulations of binary-black-hole initial data, is maximal slicing, i.e.,

$$
K \equiv 0 .
$$

Also for simplicity, we choose to make the excision surface $\mathcal{S}$ consist of coordinate spheres:

$$
\mathcal{S}=\bigcup_{a=1}^{n} \mathcal{S}_{a}
$$

where $\mathcal{S}_{a}$ are surfaces of constant Euclidean distance $r_{\text {exc }}$ about the center of each excised hole, and $n=1$ or 2 is the number of black holes present in the initial data.

The boundary condition for the lapse on $\mathcal{S}$ determines the temporal gauge; we adopt the condition given in Eq. (59a) of Ref. [28]:

$$
\frac{\partial}{\partial r_{a}}(\alpha \psi)=0 \text { on } \mathcal{S}_{a},
$$

where $r_{a}$ is the Euclidean distance from the center of hole $a$. This type of initial data is used in Refs. $[49,65,66]$.

\section{Superposed Kerr-Schild}

Single black holes with angular [50,51] or linear [52] momentum do not admit conformally flat spatial slicings; therefore, conformal flatness [Eq. (41)] is necessarily deficient. This has motivated investigations of binary-blackhole initial data whose free data have stronger physical motivation, e.g., Refs. [37,38,53-59]. 
In this subsection, we consider conformally curved data that are in the same spirit as the SKS data of Refs. [37,38] although here (i) we apply the idea to the QE-XCTS formalism, and (ii) as discussed below, our free data is very nearly conformally flat and maximally sliced everywhere except in the vicinity of the black holes.

The choices we make here generalize the conformally curved data in Chapter 6 of Ref. [39] to nonzero spins. Specifically, the free data and lapse boundary condition will be chosen so that the conformal geometry near each hole's horizon is that of a boosted, spinning, Kerr-Schild black hole. The conformal metric $\tilde{g}_{i j}$ and the mean curvature $K$ take the form

$$
\begin{gathered}
\tilde{g}_{i j}:=f_{i j}+\sum_{a=1}^{n} e^{-r_{a}^{2} / w_{a}^{2}}\left(g_{i j}^{a}-f_{i j}\right), \\
K:=\sum_{a=1}^{n} e^{-r_{a}^{2} / w_{a}^{2}} K_{a} .
\end{gathered}
$$

Here $g_{i j}^{a}$ and $K_{a}$ are the spatial metric and mean curvature, respectively, of a boosted, spinning Kerr-Schild black hole with mass $\tilde{M}_{a}$, spin $\tilde{S}_{a}$, and speed $\tilde{v}_{a}$.

Far from each hole's horizon, the conformal metric is very nearly flat; this prevents the conformal factor from diverging on the outer boundary [39]. The parameter $w_{a}$ is a weighting factor that determines how quickly the curved parts of the conformal data decay with Euclidean distance $r_{a}(a=1,2, \ldots)$ from hole $a$; in this paper, the weight factor $w_{a}$ is chosen to be larger than the size scale of hole $a$ but smaller than the distance $d$ to the companion hole (if any): $M_{a} \lesssim w_{a} \lesssim d_{a}$. This is similar to the "attenuated" superposed Kerr-Schild data of Refs. [38,60], except that here the weighting functions are Gaussians which vanish far from the holes, while in Refs. $[38,60]$ the weighting functions go to unity far from the holes.

The excision surfaces $\mathcal{S}_{a}$ are not coordinate spheres unless $\tilde{S}_{a}=0$ and $\tilde{v}_{a}=0$. Instead they are deformed in two ways. (i) They are distorted so that they are surfaces of constant Kerr radius $r_{\text {Kerr }}$, i.e.,

$$
\frac{x^{2}+y^{2}}{r_{\text {Kerr }}^{2}+\tilde{S}_{a}^{2} / \tilde{M}_{a}^{2}}+\frac{z^{2}}{r_{\text {Kerr }}^{2}}=1,
$$

where $x, y$, and $z$ are Cartesian coordinates on the $\mathcal{S}$. Then, (ii) the excision surfaces are Lorentz-contracted along the direction of the boost.

The boundary condition for the lapse $\alpha$ on $\mathcal{S}_{a}$ is a Dirichlet condition that causes $\alpha$ (and, consequently, the temporal gauge) in the vicinity of each hole to be nearly that of the corresponding Kerr-Schild spacetime, i.e.,

$$
\alpha \psi=1+\sum_{a=1}^{n} e^{-r_{a}^{2} / w_{a}^{2}}\left(\alpha_{a}-1\right) \quad \text { on } \mathcal{S}_{a},
$$

where $\alpha_{a}$ is the lapse corresponding to the Kerr-Schild spacetime $a$.

\section{SINGLE-BLACK-HOLE INITIAL DATA WITH NEARLY EXTREMAL SPINS}

In this section, we examine to which extent the formalisms presented in Sec. II can generate single-black-hole initial data with nearly extremal spin. We consider first Bowen-York initial data and then conformally flat quasi-

TABLE I. Summary of the initial-data sets constructed in this paper. The first row (BY-Single) represents Bowen-York initial data for single black holes of various spins. The next two rows (CFMS-Single and CFMS) are quasiequilibrium, conformally flat, maximally sliced initial data for single and binary spinning black holes, respectively. All other data sets employ superposed Kerr-Schild quasiequilibrium data with the second block of rows representing families of initial-data sets for various spins and the last block of rows representing individual data sets to be evolved. The data sets SKS-0.93-E0 to SKS-0.93-E3 demonstrate eccentricity removal, and

\begin{tabular}{|c|c|c|c|c|c|c|c|c|c|c|c|c|}
\hline Label & Section & Figures & $\mathrm{n}$ & $\mathrm{d}$ & $\Omega_{0}$ & $\dot{a}_{0} \times 10^{4}$ & $\Omega_{r}$ or $S / m_{p}^{2}$ & $\tilde{S}$ & $\left|\chi_{\mathrm{AKV}}\right|$ & $M_{\text {irr }}$ & $M$ & $E_{\mathrm{ADM}}$ \\
\hline BY-Single & III A & $1-5,8$, and 19 & 1 & $\cdots$ & $\cdots$ & .. & $0.01 \leq S / m_{p}^{2} \leq 10^{4}$ & $\cdots$ & & & & \\
\hline CFMS-Single & III B & $6-8$ and 19 & 1 & . & $\cdots$ & $\cdots$ & $0 \leq \Omega_{r} \leq 0.191$ & $\cdots$ & & & & \\
\hline CFMS & IVA & 9 and 13 & 2 & 32 & 0.007985 & 0 & $0 \leq \Omega_{r} \leq 0.1615$ & $\cdots$ & & & & \\
\hline SKS-0.0 & IV B & 11 and 13 & 2 & 32 & 0.006787 & 0 & $0 \leq \Omega_{r} \leq 0.24$ & 0 & & & & \\
\hline SKS-0.5 & IV B & 11 and 13 & 2 & 32 & 0.006787 & 0 & $0 \leq \Omega_{r} \leq 0.27$ & 0.5 & & & & \\
\hline SKS-0.93 & IV B & $11-13$ & 2 & 32 & 0.006787 & 0 & $0 \leq \Omega_{r} \leq 0.35$ & 0.93 & & & & \\
\hline SKS-0.99 & IV B & $10-13$ & 2 & 32 & 0.007002 & 3.332 & $0.28 \leq \Omega_{r} \leq 0.39$ & 0.99 & & & & \\
\hline SKS-0.93-E0 & VB & 14 & 2 & 32 & 0.006787 & 0 & 0.28 & 0.93 & 0.9278 & 0.9371 & 1.131 & 2.243 \\
\hline SKS-0.93-E1 & VB & 14 & 2 & 32 & 0.007 & 0 & 0.28 & 0.93 & 0.9284 & 0.9375 & 1.132 & 2.247 \\
\hline SKS-0.93-E2 & VB & 14 & 2 & 32 & 0.006977 & 3.084 & 0.28 & 0.93 & 0.9275 & 0.9395 & 1.134 & 2.249 \\
\hline SKS-0.93-E3 & $\mathrm{VC}$ & $10,11,13-16$, and 19 & 2 & 32 & 0.007002 & 3.332 & 0.28 & 0.93 & 0.9275 & 0.9397 & 1.134 & 2.250 \\
\hline SKS-Headon & VD & $10,11,13$, and $17-19$ & 2 & 100 & 0 & 0 & 0.3418 & 0.97 & 0.9701 & 0.8943 & 1.135 & 2.257 \\
\hline
\end{tabular}
SKS-Headon is used in a head-on evolution. The first block of columns gives the label used for each data set and the relevant section of this paper devoted to it. The next block of columns lists the most important parameters entering the initial data. The last block of columns lists some properties of those data sets that we evolve in Sec. V. 
equilibrium data. Since superposed Kerr-Schild data can represent single Kerr black holes exactly, there is no need to investigate single-hole superposed Kerr-Schild data. In Sec. IV, we will both consider conformally flat and superposed Kerr-Schild data for binary black holes.

To orient the reader, the initial-data sets constructed in this section, as well as the binary-black-hole data sets constructed in Sec. IV, are summarized in Table I.

Unless noted otherwise, all spins presented in this section are measured using the approximate-Killing-vector spin $\chi_{\mathrm{AKV}}$ described in Appendix A. Therefore, the subscript "AKV" in $\chi_{\mathrm{AKV}}$ will be suppressed for simplicity.

\section{A. Bowen-York (puncture) initial data}

As discussed in Sec. II B, for a single spinning black hole at rest, puncture initial data are identical to inversionsymmetric initial data. Such solutions have been examined in the past (e.g., $[48,61]$ ), and additional results were obtained (partly in parallel to this work) in the study by Dain, Lousto, and Zlochower [25].

We revisit this topic here to determine the maximum possible spin of Bowen-York initial data more accurately than before, to establish the power-law coefficients for the approach to these limits with increasing spin parameter $S$, and to present new results about the geometric structure of Bowen-York (BY) initial data with a very large spin parameter.

We solve Eq. (30) with the pseudospectral elliptic solver described in Ref. [62]. The singular point of $u$ at the origin is covered by a small rectangular block extending from $\pm 10^{-4} m_{p}$ along each coordinate axis. This block overlaps four concentric spherical shells with radii of the boundaries at $8 \times 10^{-5} m_{p}, 0.005 m_{p}, 0.3 m_{p}, 50 m_{p}$, and $10^{9} m_{p}$. The equations are solved at several different resolutions, with the highest resolution using $20^{3}$ basis functions in the cube, $L=18$ in the spheres and 26 and 19 radial basis functions in the inner and outer two spherical shells, respectively.

Because of the axisymmetry of the data set, the rotational Killing vector of the apparent horizon is simply $\partial_{\phi}$. The integral for the quasilocal spin Eq. (A1) turns out to be independent of $\psi$ and can be evaluated analytically with a result equal to the spin parameter $S$. Thus we can use this initial-data set to check how well our spin diagnostics and our ADM angular momentum diagnostic works (recall that $J_{\mathrm{ADM}}$ is also equal to the spin parameter $S$ ). This comparison is performed in Fig. 1, which shows relative differences between the numerically extracted values for the AKV spin, the coordinate spin (defined with the AKV spin in Appendix A), and the $\mathrm{ADM}$ angular momentum $J_{\mathrm{ADM}}$ relative to the expected answer $S$. The figure also shows differences between neighboring resolutions for the two quantities of interest below: $S / M^{2}=\chi$ and $S / E_{\mathrm{ADM}}^{2}=$ $J_{\mathrm{ADM}} / E_{\mathrm{ADM}}^{2}=\varepsilon_{J}$.

Figure 1 seems to show exponential convergence with increased resolution $N$. Since puncture data are only $C^{2}$ at

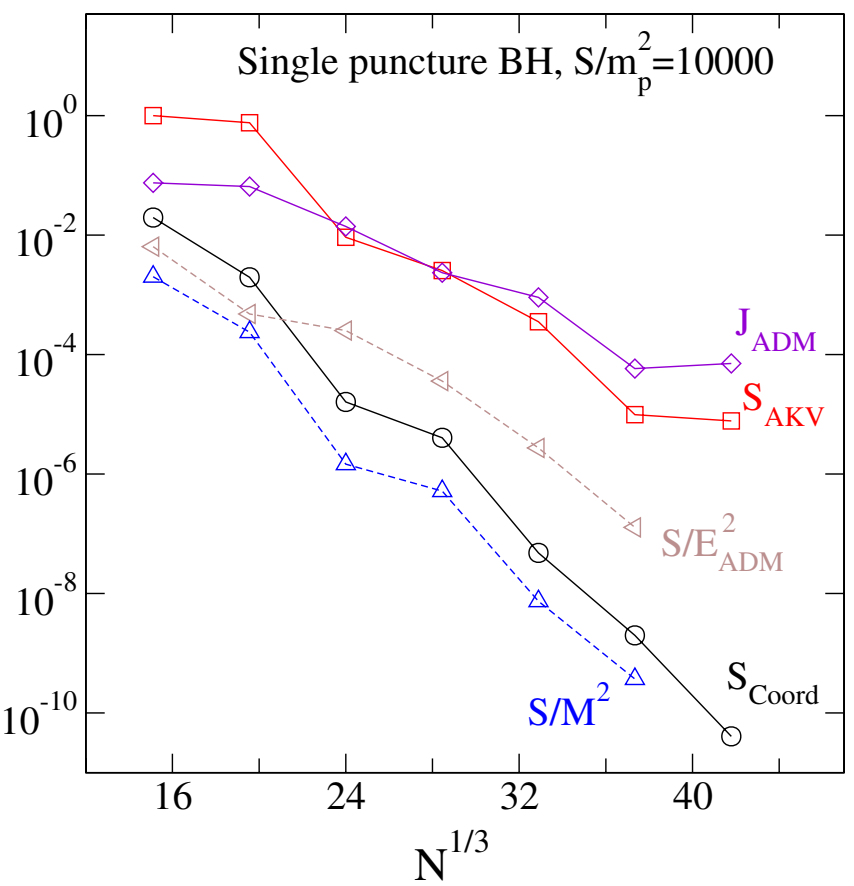

FIG. 1 (color online). Convergence test for a single puncture black hole with a very large spin parameter $S / m_{p}^{2}=10000$. Plotted are results vs resolution $N$, which is the total number of basis functions. The solid lines show the relative differences of three angular momentum measures to the analytically expected value 10000 . The dashed lines show differences from the nexthigher resolution of two dimensionless quantities for which no analytic answer is available.

the puncture, one would rather expect polynomial convergence. The effect of the nonsmoothness at the puncture is mitigated by choosing a very high resolution close to the puncture (a small cube with sides $\pm 10^{-4} m_{p}$ with $20^{3}$ basis functions). Therefore, for the resolutions considered in Fig. 1, the truncation error is dominated by the solution away from the puncture, and exponential convergence is visible. If we used infinite-precision arithmetic and were pushing toward higher resolution than shown in Fig. 1, then we would expect to eventually see polynomial convergence dominated by the cube covering the puncture.

Next, we construct a series of initial-data sets with increasing spin parameter $S$ and compute $\chi, \varepsilon_{J}$, and $\zeta$ for each initial-data set. The results are plotted in Fig. 2 and confirm earlier results [26,61]. In addition, the inset shows that the asymptotic values $\chi_{\max }=0.9837$ and $\varepsilon_{u, \max }=0.928200$ are approached as power laws in the spin parameter:

$$
\begin{aligned}
& \chi_{\max }-\chi \propto\left(\frac{S}{m_{p}^{2}}\right)^{-0.75}, \\
& \varepsilon_{J, \max }-\varepsilon_{J} \propto\left(\frac{S}{m_{p}^{2}}\right)^{-1.4} .
\end{aligned}
$$




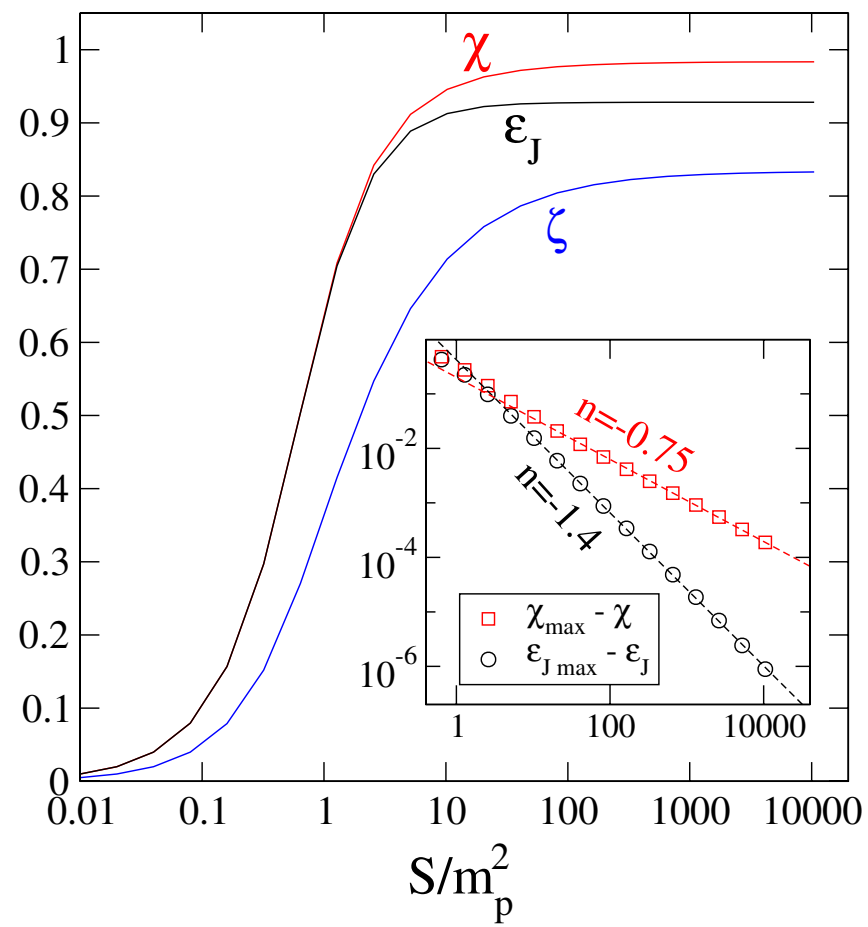

FIG. 2 (color online). Properties of single, spinning puncture black holes with spin parameter $S$ and puncture mass $m_{p}$. The dimensionless spin $\chi:=S / M^{2}$, ADM angular momentum $\varepsilon_{J}:=$ $J_{\mathrm{ADM}} / E_{\mathrm{ADM}}^{2}$, and spin-extremality parameter $\zeta:=S /\left(2 M_{\mathrm{irr}}^{2}\right)$ are plotted against the spin parameter $S / m_{p}^{2}$. The horizon mass $M$ is related to the spin $S$ and irreducible mass $M_{\text {irr }}$ in Eq. (2).

The exponents of these power laws are computed here for the first time.

To confirm that the apparent horizon is indeed at $r=$ $R_{\text {inv }}$, we ran our apparent horizon finder on the high-spin puncture initial-data sets. The horizon finder had great difficulty converging, and the reason for this becomes clear from Fig. 3. The main panel of this figure shows the area of spheres with coordinate radius $r$. The area is minimal at $r=m_{p} / 2$, as it must be, since $m_{p} / 2=R_{\text {inv }}$ is the radius of the inversion sphere. However, the area is almost constant over a wide range in $r$ - for $S / m_{p}^{2}=10000$ over about two decades in either direction: $0.01 \lesssim r / R_{\text {inv }} \leqslant 100$. Thus, the Einstein-Rosen bridge (the throat) connecting the two asymptotically flat universes lengthens as the spin increases, giving rise to an ever-lengthening cylinder. If this were a perfect cylinder, then the expansion would be zero for any $r=$ const cross section. Because the geometry is not perfectly cylindrical, the expansion vanishes only for $r=m_{p} / 2=R_{\text {inv }}$, but remains very small even a significant distance away from $r=m_{p} / 2=R_{\mathrm{inv}}$. This is shown in the inset, which plots the residual of the apparent horizon finder at different radii.

With the lengthening of the throat, the interval in $r$ with small expansion lengthens, and the value of the expansion within this interval reduces. Both effects make it harder for

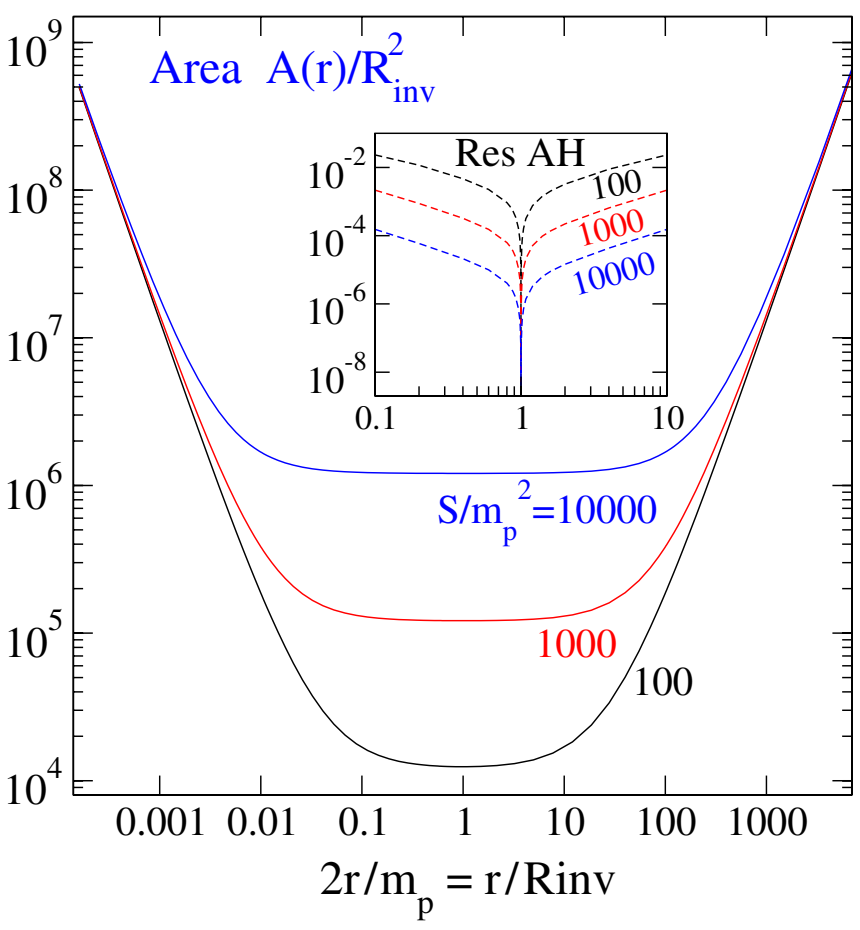

FIG. 3 (color online). Properties of coordinate spheres with radius $r$ for high-spin puncture initial data. Main panel: Area of these spheres. Inset: Residual of the apparent horizon equation on these spheres. The area is almost constant over several orders of magnitude in $r$. The apparent-horizon residual vanishes at $r=$ $R_{\text {inv }}$ but is very small over a wide range of $r$.

the apparent horizon finder to converge. In Fig. 2, we have used our knowledge of the location of the apparent horizon to set $r_{\mathrm{AH}}=m_{p} / 2$ rather than to find this surface numerically. Without this knowledge, which arises due to the identification of puncture data and inversion-symmetric data, computation of Fig. 2 would have been significantly harder, perhaps impossible.

Let us assume for the moment that the solution $\psi(r)=$ $\frac{m_{p}}{2 r}+1+u(r)$ is spherically symmetric (we give numerical evidence below that this is indeed a good approximation). Because $g_{i j}=\psi^{4} f_{i j}$, the area of coordinate spheres is then given by

$$
A(r)=4 \pi \psi^{2}(r) r .
$$

In the throat region, where $A(r) \approx$ const, the conformal factor must therefore behave like $1 / \sqrt{r}$, as also argued independently by Dain, Lousto, and Zlochower [25].

To extend on Dain, Lousto, and Zlochower's analysis, let us substitute Eq. (23) into Eq. (24) to obtain the wellknown equation

$$
\tilde{\nabla}^{2} \psi=-\frac{9 S^{2} \sin ^{2} \theta}{4 r^{6}} \psi^{-7},
$$

where $\theta$ is the angle between the spin direction and the point $x^{i}$. Continuing to assume that $\psi$ is approximately 
spherically symmetric, we can replace the factor $\sin ^{2} \theta$ by its angular average $(4 \pi)^{-1} \int \sin ^{2} \theta d \Omega=2 / 3$ and obtain

$$
\frac{d^{2} \bar{\psi}}{d r^{2}}+\frac{2}{r} \frac{d \bar{\psi}}{d r}=-\frac{3 S^{2}}{2 r^{6}} \bar{\psi}^{-7} .
$$

Here, we introduced an overbar $\bar{\psi}$ to distinguish the spherically symmetric solution $\bar{\psi}(r)$ of Eq. (53) from the full solution $\psi\left(x^{i}\right)$ of puncture/inversion-symmetric initial data. Following Dain, Lousto, and Zlochower [25] we assume that the conformal factor behaves as a power law $\left[\bar{\psi}(r)=A r^{\alpha}\right]$ and substitute this into Eq. (53). We find that Eq. (53) determines the power-law exponent $\alpha=-1 / 2$ and the overall amplitude $A=\left(6 S^{2}\right)^{1 / 8}$, so that

$$
\bar{\psi}(r)=\frac{\left(6 S^{2}\right)^{1 / 8}}{\sqrt{r}}=96^{1 / 8}\left(\frac{S}{m_{p}^{2}}\right)^{1 / 4}\left(\frac{r}{R_{\mathrm{inv}}}\right)^{-1 / 2} .
$$

In Eq. (54), we chose the scaling $S / m_{p}^{2}$, which is commonly used in the puncture-data literature, but kept $r / R_{\mathrm{inv}}$ to emphasize the inversion symmetry of the data in our figures (in a log-plot using $r / R_{\text {inv }}$, the solution will appear symmetric; see, e.g., Fig. 3$)$. While $\bar{\psi}(r)$ solves the spherically symmetric Eq. (53) exactly, it must deviate from $\psi\left(x^{i}\right)$ for sufficiently large $r$ because $\bar{\psi} \rightarrow 0$ as $r \rightarrow \infty$, whereas $\psi \rightarrow 1$. The deviation will become significant when $\bar{\psi} \sim 1$, i.e., at radius $r_{x} \sim \sqrt{S / m_{p}^{2}}$. Because of inversion symmetry, this implies a lower bound of validity at $1 / r_{x}$, so that Eq. (54) holds for

$$
\left(\frac{S}{m_{p}^{2}}\right)^{-1 / 2} \lesssim \frac{r}{R_{\mathrm{inv}}} \lesssim\left(\frac{S}{m_{p}^{2}}\right)^{1 / 2} .
$$

The circumference of the cylindrical throat is

$$
\mathcal{C}=2 \pi \bar{\psi}(r)^{2} r=2 \pi 96^{1 / 4} \sqrt{\frac{S}{m_{p}^{2}}} R_{\text {inv }}
$$

and its length is

$$
\mathcal{L}=\int_{\left(S / m_{p}^{2}\right)^{-1 / 2}}^{\left(S / m^{2}\right)^{1 / 2}} \bar{\psi}^{2}(r) d r=96^{1 / 4} \sqrt{\frac{S}{m_{p}^{2}}} \ln \left(\frac{S}{m_{p}^{2}}\right) R_{\mathrm{inv}} .
$$

Therefore, the ratio of length to circumference

$$
\frac{\mathcal{L}}{\mathcal{C}}=\frac{1}{2 \pi} \ln \left(\frac{S}{m_{p}^{2}}\right)
$$

grows without bound as $S / \mathrm{m}_{p}^{2}$ becomes large, albeit very slowly. The scaling with $\left(S / m_{p}^{2}\right)^{1 / 2}$ in Eqs. (55)-(57) might seem somewhat surprising. However, in the large spin limit, $S / M^{2}$ is just a constant close to unity (namely, $\chi_{\max }=0.9837$ ). Therefore, $S^{1 / 2} \approx M$, i.e., the scaling $S^{1 / 2}$ is effectively merely a scaling with mass.

Figure 4 shows the conformal factor $\psi$, the "puncture function" $u$, and the estimate $\bar{\psi}$ of Eq. (54) for three

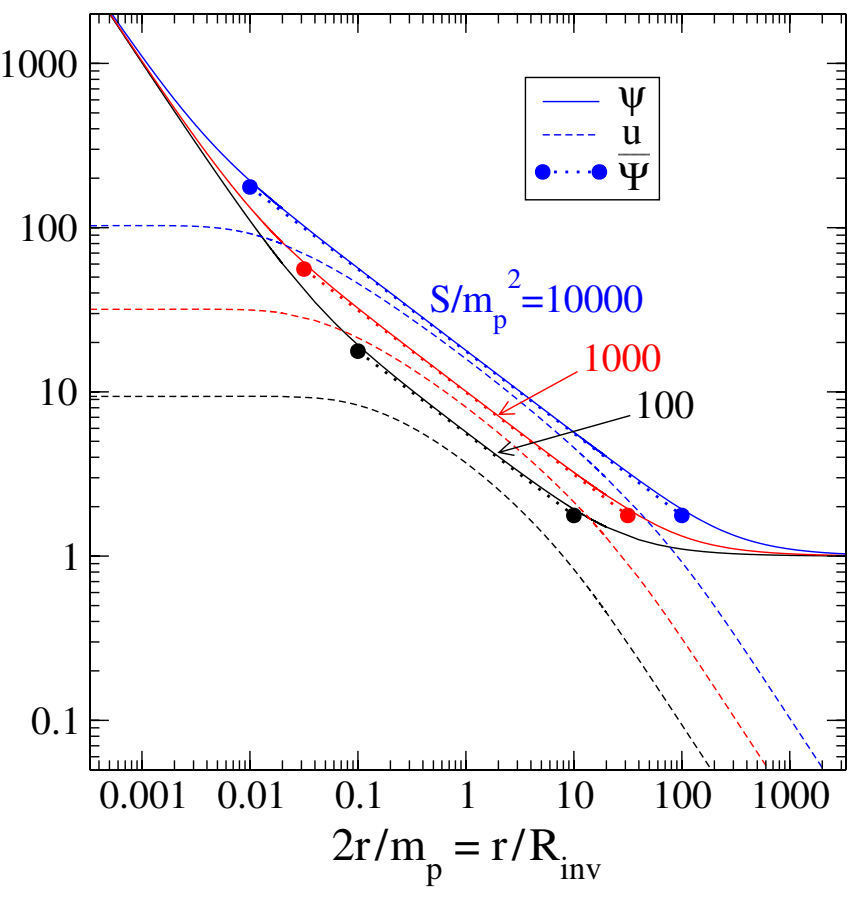

FIG. 4 (color online). Solutions of high-spin puncture initial data. Plotted are the conformal factor $\psi$ and puncture function $u$ in the equatorial plane as a function of radius $r$. Furthermore, the approximate solution $\bar{\psi}$ is included, with solid circles denoting the range of validity of this approximation; cf. Eq. (55). Three curves each are plotted, corresponding from top to bottom to $S / m_{p}^{2}=10000,1000$, and 100 .

different values of $S / \mathrm{m}_{p}^{2}$. There are several noteworthy features in this figure. First, both $\psi$ and $u$ show clearly three different regimes:

(i) For large $r, \psi \approx 1$ and $u \propto 1 / r$. This is the upper asymptotically flat end.

(ii) For intermediate $r, \psi \propto 1 / \sqrt{r}$ and $u \propto 1 / \sqrt{r}$. This is the cylindrical geometry extending symmetrically around the throat. This region becomes more pronounced as $S$ increases.

(iii) For small $r, \psi \propto 1 / r$ and $u \approx$ const. This is the lower asymptotically flat end.

Figure 4 also plots the approximate solution $\bar{\psi}$ [cf. Eq. (54)] for its range of validity [given by Eq. (55)]. Note that slope and amplitude of $\bar{\psi}$ fit very well the numerical solution $\psi$. In fact, the agreement is much better than with $u$.

One could also have started the calculation that led to Eq. (54) with Eq. (30). Assuming spherical symmetry, and assuming that $u \gg m_{p} /(2 r)+1$, we would have derived Eq. (53), but with $\bar{\psi}$ replaced by $u$. We would then have found the approximate behavior Eq. (54) for $u$. The disadvantage of this approach is the need for additional approximations, which reduce the accuracy of the result. From Fig. 4 we see that, in the throat region, the dotted 
lines representing $\bar{\psi}$ are close to the dashed lines of $u$. But the agreement between $\psi$ and $\bar{\psi}$ is certainly better.

Finally, we note that the limits of validity of $\bar{\psi}$ [Eq. (55)] match very nicely the points where the numerical $\psi$ diverges from $\bar{\psi}$.

To close this section, we present numerical evidence that indeed $\psi$ is approximately spherically symmetric, the assumption that entered into our derivation of Eq. (54). We decompose the conformal factor of the numerical puncture-data solutions into spherical harmonics,

$$
\psi(r, \theta, \phi)=\sum_{l=0}^{\infty} \sum_{m=-l}^{l} \psi_{l m}(r) Y_{l m}(\theta, \phi),
$$

and plot in Fig. 5 the sizes of the $l \neq 0$ modes relative to the spherically symmetric mode $\psi_{00}$. Because of the symmetries of the problem, the only nonzero modes have $m=$ 0 and even $l$. In the throat region, the largest nonspherically symmetric mode $\psi_{20}$ is about a factor of 65 smaller than the spherically symmetric mode. With increasing $l, \psi_{l m}$ decays very rapidly. Also, in both asymptotically flat ends, the non-spherically symmetric modes decay more rapidly than the $l=0$ mode, as expected for asymptotically flat data. This figure again shows nicely the inversion symmetry of the data, under $r / R_{\mathrm{inv}} \rightarrow$ $\left(r / R_{\text {inv }}\right)^{-1}$. Given the simple structure of the higher modes, it should be possible to extend the analytical analysis of the throat to include the nonspherical contributions. To do so, one would expand $\psi$ as a series in Legendre polynomials in $\theta$; the $\psi^{-7}$ term on the right-hand side of Eq. (52) would result in a set of ordinary differential equations for those

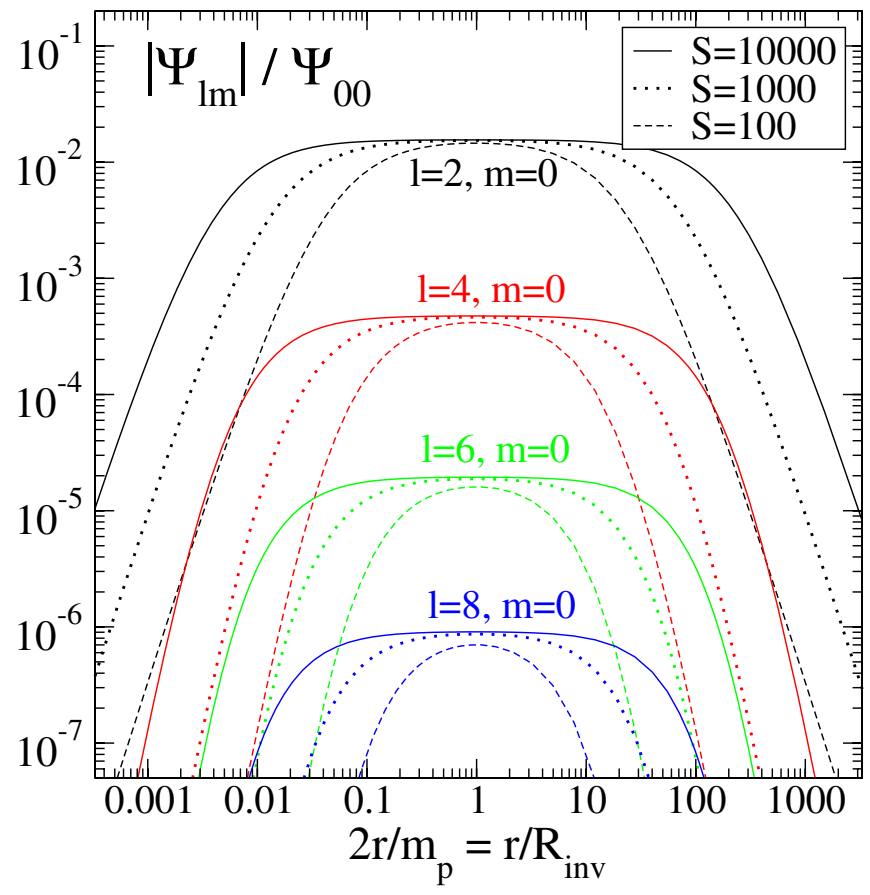

FIG. 5 (color online). Angular decomposition of the conformal factor $\psi(r, \theta, \phi)$ for single-black-hole puncture data. coefficients. In the throat region, the radial behavior of each mode should be $\propto 1 / \sqrt{r}$, and the ordinary differential equations should simplify to algebraic relations.

\section{B. Quasiequilibrium extended-conformal-thin- sandwich data}

We have seen in Sec. III A that puncture initial data for single, spinning black holes can be constructed for holes with initial spins of $\chi \leq 0.9837$. In this section, we address the analogous question for excision black-hole initial data: How rapid can the initial spin be for a single, spinning black hole constructed using QE-XCTS initial data?

As noted previously, if the free data $\tilde{g}_{i j}$ and $K$ are chosen to agree with the analytic values for a Kerr black hole, $g_{i j}^{\text {Kerr }}$ and $K^{\text {Kerr }}$, then the QE-XCTS initial data can exactly represent a single Kerr black hole. In this case, $\chi=1$ is obtained trivially by choosing $\tilde{S}=\tilde{M}^{2}=1$, where $\tilde{M}$ and $\tilde{S}$ are the mass and spin, respectively, of the Kerr black hole described by the conformal metric.

Setting aside this trivial solution, we construct CFMS data for a single, spinning hole. We construct a family of QE-XCTS initial-data sets for single spinning black holes by numerically solving the XCTS equations [in the form stated in Eqs. (37a)-(37c)] using the same spectral elliptic solver [62] as in Sec. III A. The free data are given by Eqs. (41) and (42) and by Eqs. (36a) and (36b).

On the outer boundary $\mathcal{B}$, we impose Eqs. (38a)-(38c). So that the coordinates are asymptotically inertial, we choose $\Omega_{0}=\dot{a}_{0}=0$ in Eq. (38c).

We excise a coordinate sphere of radius $r_{\mathrm{exc}}$ about the origin, where

$$
r_{\mathrm{exc}}=0.85949977
$$

is chosen such that for zero spin $M=1$. On this inner boundary $\mathcal{S}$, we impose Eqs. (39), (40), and (44). The spin is determined by Eq. (40): First, the vector $\xi^{i}$ is chosen to be the coordinate rotation vector $\partial_{\phi}$, making the spin point along the positive $z$ axis; then, the rotation parameter $\Omega_{r}$ is varied while the other parameters are held fixed. The spin is measured on the apparent horizon using the approximate-Killing-vector spin (Appendix A); because in this case the space is axisymmetric, the "approximate" Killing vector reduces to the corresponding exact rotational Killing vector.

Figure 6 show how the mass $M$ and AKV spin $S$ depend on $\Omega_{r}$. At $\Omega_{r}=0$, we find the spherically symmetric solution with $S=0$ and $M_{\text {irr }}=M=1$ [the mass is proportional to the excision radius, and Eq. (60) sets it to unity]. Using this spherically symmetric solution as an initial guess for the elliptic solver, we find solutions for increasing $\Omega_{r}$ with spin increasing initially linearly with $\Omega_{r}$ and with approximately constant mass. Beyond some critical $\Omega_{r \text {,crit }}$, the elliptic solver fails to converge, and close to this point, all quantities vary in proportion to 
$\sqrt{\Omega_{r \text {,rit }}-\Omega_{r}}$. These symptoms indicate a critical point where the solutions "turn over" and continue towards smaller $\Omega_{r}$. Analogous nonunique solutions of the XCTS equations have been discovered before in Ref. [34]. To construct solutions along the upper branch, one must choose a sufficiently close initial guess for the elliptic solver; we follow the steps outlined in Ref. [34] and are able to find solutions along the upper branch for a wide range of $\Omega_{r}<\Omega_{r \text {,crit }}$. As Fig. 6 shows, mass and spin of the horizon in solutions along the upper branch increase with decreasing $\Omega_{r}$, analogous to the findings in [34,35].

Figure 7 shows the dependence of $\chi=S / M^{2}, \varepsilon_{J}=$ $J_{\mathrm{ADM}} / E_{\mathrm{ADM}}^{2}$, and $\zeta=S /\left(2 M_{\mathrm{irr}}^{2}\right)$ on $\Omega_{r}$. The curves reflect again the nonunique solutions. The dimensionless spin $\chi$ increases continuously along the lower branch and reaches $\chi \approx 0.85$ at the critical point. As $\Omega_{r}$ is decreased along the upper branch, $\chi$ continues to increase, eventually reaching values larger than 0.99 . It appears $\chi$ continues to increase as $\Omega_{r} \rightarrow 0$. To find the limiting value, consider that the behavior of the extremality parameter $\zeta$ in the inset of Fig. 7. Assuming that $\zeta$ can be extrapolated to $\Omega_{r} \rightarrow 0$, we find a limiting value of $\zeta \approx 0.88$. By Eq. (9), this implies a maximal value of $\chi \approx 0.992$.

In Figs. 6 and 7, the data sets on the lower branch appear to be physically reasonable. For spins $\chi \lesssim 0.85$, the mass

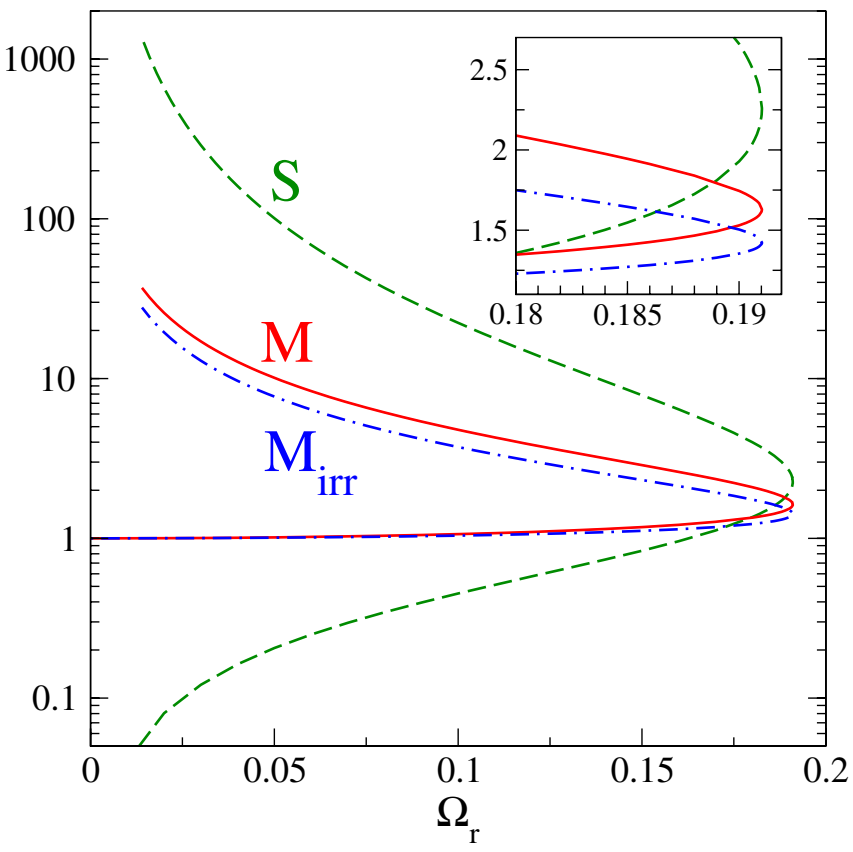

FIG. 6 (color online). Conformally flat, maximally sliced, quasiequilibrium initial-data sets with a single, spinning black hole. We plot the horizon mass $M$, irreducible mass $M_{\text {irr }}$, and the (approximate-Killing-vector) spin $S$ against the rotation parameter $\Omega_{r}$ [cf. Eq. (40)]. Only $\Omega_{r}$ is varied in this figure; all other parameters are held fixed. The upper and lower points with the same $\Omega_{r}$ are obtained numerically by choosing different initial guesses. The inset shows a close-up view of the turning point, which occurs at $\Omega_{r} \approx 0.191$.

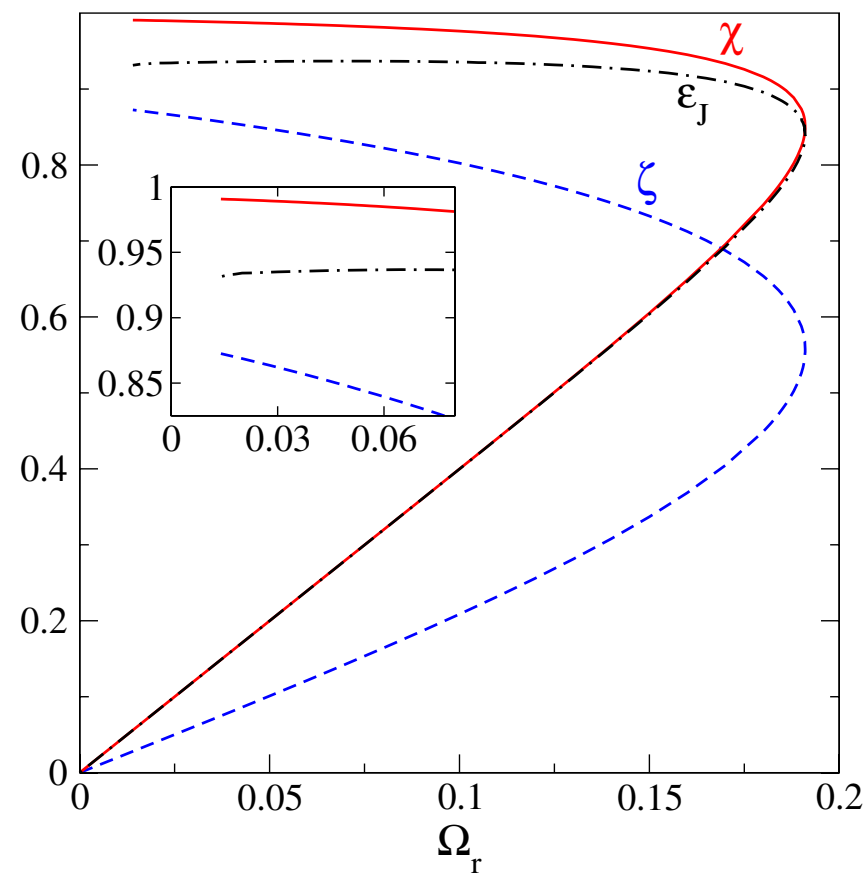

FIG. 7 (color online). Conformally flat, maximally sliced quasiequilibrium initial-data sets with a single spinning black hole: The dimensionless spin $\chi$, dimensionless ADM angular momentum $\varepsilon_{J}$, and spin-extremality parameter $\zeta$ plotted against $\Omega_{r}$ [cf. Eq. (40)]. Only $\Omega_{r}$ is varied in this figure; all other parameters are held fixed. The inset enlarges the area in the upper left corner; we are able to generate data sets with $\chi>0.99$, whereas the largest spin obtainable on the lower branch is $\chi \approx 0.85$.

$M$ is nearly constant, and the dimensionless spin $\chi$ increases linearly with $\Omega_{r}$. Furthermore, as $\Omega_{r} \rightarrow 0$ the lower branch continuously approaches the exact Schwarzschild spacetime (see [28]). The upper branch appears to be physically less reasonable; for instance, the spin $\chi$ increases for decreasing horizon frequency $\Omega_{r}$. Comparing Figs. 2 and 7, we see that the QE-XCTS data lead to somewhat larger values of $\chi$ and $\varepsilon_{J}$ relative to puncture data. However, the values are not too different, and similar trends remain. For instance, $\chi$ is much closer to unity than $\varepsilon_{J}$.

To investigate differences or similarities between puncture data and QE-XCTS data further, we compute embedding diagrams of the equatorial planes of these data sets. The initial data for single black holes have rotational symmetry about the $z$ axis, so the metric (12) on the initial-data hypersurface, when restricted to the equatorial plane, can be written as

$$
d s^{2}=\psi^{4}\left(d r^{2}+r^{2} d \phi^{2}\right)
$$

where $r$ and $\phi$ are the usual polar coordinates. This metric is now required to equal the induced metric on the $2 \mathrm{D}$ surface given by $Z=Z(R)$ embedded in a 3D Euclidean space with line element 


$$
d s_{\text {Euclidean }}^{2}=d R^{2}+R^{2} d \phi^{2}+d Z^{2} .
$$

Setting $d Z=\frac{d Z}{d R} d R$, we obtain the induced metric on the $Z=Z(R)$ surface

$$
d s^{2}=\left[1+\left(\frac{d Z}{d R}\right)^{2}\right] d R^{2}+R^{2} d \phi^{2} .
$$

Equating Eqs. (61) and (63), we find

$$
R=\psi^{2} r
$$

and

$$
\left[1+\left(\frac{d Z}{d R}\right)^{2}\right] d R^{2}=\psi^{4} d r^{2} .
$$

Combining (64) and (65) results in

$$
\left(\frac{d Z}{d r}\right)^{2}=-4 r \psi^{2} \frac{d \psi}{d r}\left(\psi+r \frac{d \psi}{d r}\right) .
$$

Since the pseudospectral elliptic solver gives $\psi$ as a function of $r$, Eqs. (64) and (66) allow us to solve for the embedding radius $R$ and the embedding height $Z$ in terms of $r$.

Figure 8 shows embedding diagrams for three sets of QE-XCTS and puncture data. We have set $Z=0$ at $r=$ $r_{\text {exc }}$ for QE-XCTS data and at $r=R_{\text {inv }}$ for puncture data. This figure also contains the embedding of a plane through Schwarzschild in Schwarzschild coordinates (i.e., the $S=$

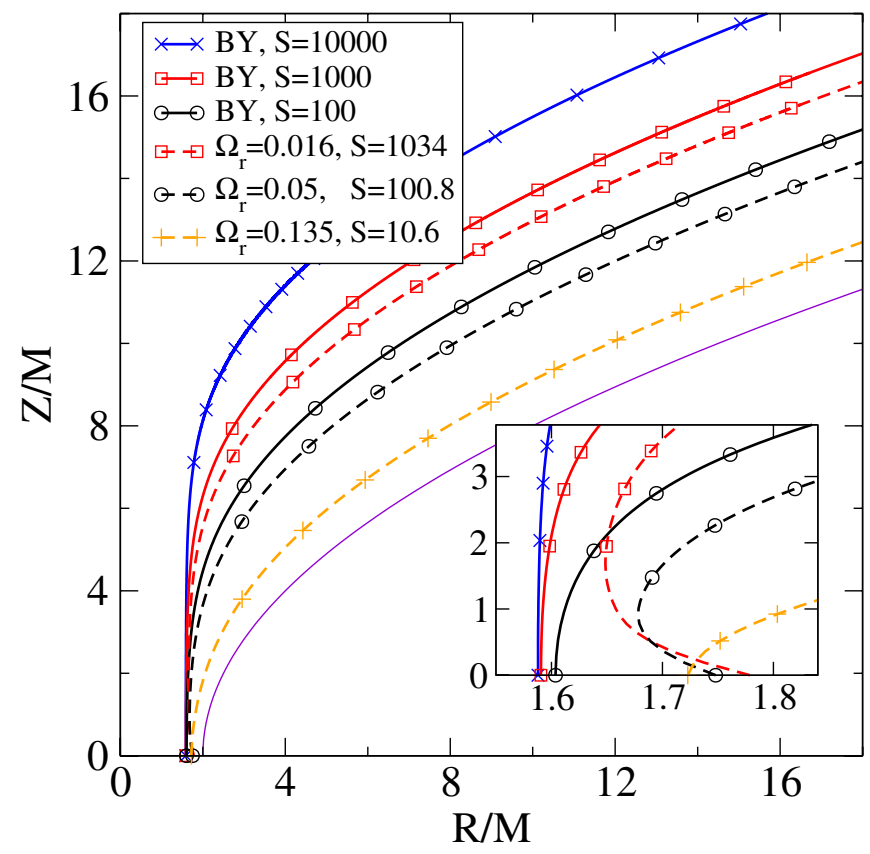

FIG. 8 (color online). Embedding diagrams for puncture and quasiequilibrium initial data. Plotted is the embedding height $Z$ as a function of the embedding radius $R$, both scaled by the mass $M$. For quasiequilibrium data (dashed lines), $Z=0$ at $r=r_{\text {exc }}$; for puncture data (solid lines), $Z=0$ at $r=R_{\text {inv }}$. The thin solid purple curve represents the embedding of a plane through a Schwarzschild black hole in Schwarzschild slicing.

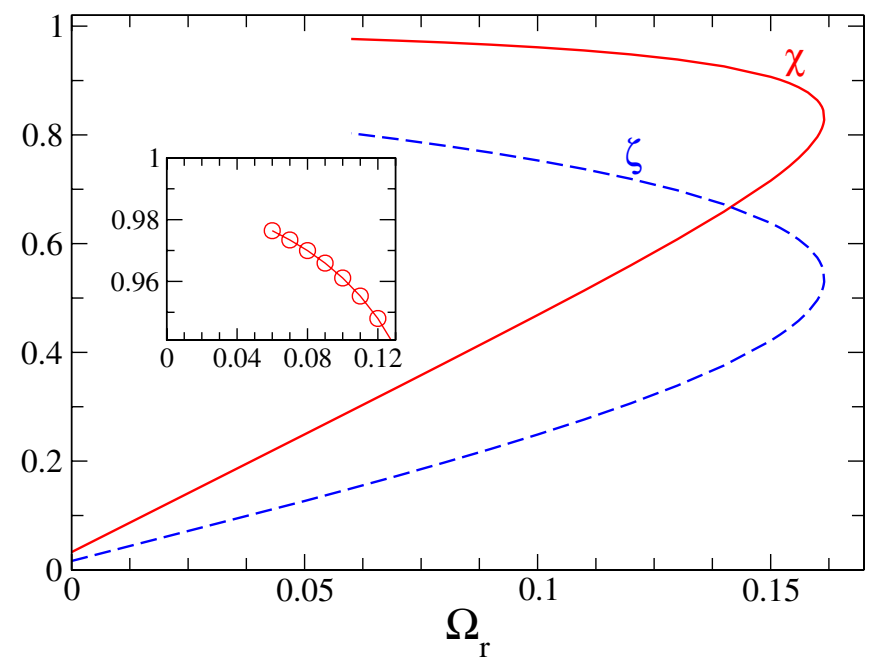

FIG. 9 (color online). Main panel: Dimensionless spin $\chi$ [Eq. (1)] and spin-extremality parameter $\zeta$ [Eq. (8)] for the family CFMS of spinning binary-black-hole initial data. Inset: Enlargement of $\chi$ toward the end of the upper branch, with circles denoting the individual initial-data sets that were constructed. Compare with Fig. 7.

0 limit of BY puncture data), given by $R / M=$ $Z^{2} /\left(8 M^{2}\right)+2$. Both puncture data and CFMS data exhibit a lengthening throat with increasing spin $S / M^{2}$. For puncture data, this lengthening can be deduced from the analytical results in Sec. III A: As the spin parameter $S$ of the puncture data increases by a factor of 10 while $m_{p} \equiv 1$ is held constant, we find from Eq. (57) that $\mathcal{L} / S^{1 / 2}$ should increase by

$$
\Delta \mathcal{L} / S^{1 / 2}=\frac{96^{1 / 4}}{2} \ln 10 \approx 3.60
$$

where the factor $1 / 2$ arises because $R_{\text {inv }}=m_{p} / 2=0.5$. The embedding diagram shows only the top half of the throat, and $S^{1 / 2} \approx M$ [cf. the discussion after Eq. (58)]. Therefore in Fig. 8 the $S=100,1000$, and 10000 lines for $\mathrm{BY}$ (puncture) data should be spaced by $\Delta Z / M \approx 1.80$ for large $R / M$. This indeed is the case.

The CFMS data sets appear to scale proportionally to $\sqrt{S}$, which is similar to the puncture data's behavior. Furthermore, the CFMS initial-data sets also develop a lengthening throat as $S$ becomes large (the effect is not as pronounced as for puncture data, owing to the smaller maximal $S$ we achieved). Thus it appears that large spin CFMS data might be similar to large spin puncture data. However, the throats of the QE-XCTS data show a bulge near the bottom, because for these data sets $R$ actually decreases with $r$ in the immediate vicinity of $r_{\text {exc }}$. This is unlike the puncture data, which very clearly exhibit cylindrical throats, consistent with the discussion leading to (58). 


\section{BINARY-BLACK-HOLE INITIAL DATA WITH NEARLY EXTREMAL SPINS}

In this section, we construct binary-black-hole initial data with rapid spins, confining our attention to the special case of spins aligned with the orbital angular momentum. In the limit of large separation, binary-black-hole puncture initial data will behave like two individual puncture initialdata sets. Specifically, we expect that it should be possible to construct puncture binary-black-hole initial data with initial spins $\chi(t=0) \lesssim 0.98$, but the spins will rapidly drop to $\chi \lesssim 0.93$ as the black holes settle down. For this reason, and also because puncture data are not well-suited to our pseudospectral evolution code, we will restrict our attention to binary black holes constructed with the QEXCTS approach.

As laid out in Table I, we first construct a family (labeled CFMS) of standard conformally flat initial data on maximal slices; then, we turn our attention to families (labeled SKS) of superposed Kerr-Schild initial data. Finally, we construct a few individual SKS-initial-data sets which we evolve in Sec. V. All of the data sets represent equal-mass, equal-spin black holes with spins parallel to the orbital angular momentum.

In this section, unless otherwise indicated, all dimensionless spins are the approximate-Killing-vector spin $\chi_{\mathrm{AKV}}$ (Appendix A), and the subscript AKV will be suppressed for simplicity.

\section{A. Conformally flat, maximal slicing data}

To construct conformally flat binary-black-hole data, we solve the same equations and boundary conditions as for the single-black-hole case, as described in Sec. III B, with the main difference being that we excise $t w o$ spheres with radius $r_{\text {exc }}$ [cf. Eq. (60)] with centers on the $x$ axis at $x=$ $\pm d / 2$. The initial spins of the holes are set by adjusting $\Omega_{r}$, just as in the single-hole case. The parameters $\boldsymbol{\Omega}_{\mathbf{0}}$ and $\dot{a}_{0}$ in the outer boundary condition on the shift [Eq. (38c)] determine the initial angular and radial motion of the holes, which in turn determine the initial eccentricity $e$ of the orbit. We set $\boldsymbol{\Omega}_{\mathbf{0}}=\Omega_{0} \mathbf{e}_{z}$, where $\mathbf{e}_{z}$ is a unit vector that points along the positive $z$ axis. For the CFMS family of data sets considered here, we use values for $\Omega_{0}$ and $\dot{a}_{0}$ that should result in closed, fairly circular orbits, since our choices of $\Omega_{0}$ and $\dot{a}_{0}$ lead to data sets that approximately satisfy the Komar-mass condition $E_{\mathrm{ADM}}=M_{K}$ (cf. [29]). Specifically, on the lower branch of the resulting nonunique family of initial data,

$$
\frac{\left|E_{\mathrm{ADM}}-M_{K}\right|}{E_{\mathrm{ADM}}} \lesssim 1 \% \text {, }
$$

where the Komar mass is defined by [e.g., Eq. (35) of Ref. [29]]

$$
M_{K}:=\frac{1}{4 \pi} \oint_{\infty}\left(\nabla_{i} \alpha-\beta^{j} K_{i j}\right) d A .
$$

(On the upper branch, $E_{\mathrm{ADM}}$ and $M_{K}$ differ by up to $3 \%$.)
As the rotation parameter $\Omega_{r}$ is varied (with the coordinate separation $d$ held fixed), we find that the CFMS family of binary-black-hole initial data behaves qualitatively similarly to the analogous single-black-hole initial data discussed in Sec. III B. There is a maximal $\Omega_{r \text {,crit }}$ such that no solutions can be found for $\Omega_{r}>\Omega_{r \text {,crit }}$; for values of $\Omega_{r}$ below $\Omega_{r \text {, crit }}$, two solutions exist. Figure 9 plots the dimensionless spin $\chi$ and the spin-extremality parameter $\zeta$ against $\Omega_{r}$ for this family of initial data. We only show values for one of the holes, since the masses and spins are equal. Spins larger than $\chi \approx 0.85$ appear on the upper branch. The highest spin we have been able to construct is larger than $\chi=0.97$.

\section{B. Superposed Kerr-Schild data}

In this section, we solve the same equations and boundary conditions as in the conformally flat case, except that we use SKS free data (Sec. II C 2) instead of conformally flat free data. To construct the individual Kerr-Schild data, we need to choose for each black hole the coordinate location of its center, its conformal mass $\tilde{M}$, conformal spin $\tilde{S}$, and its boost velocity. We center the black holes on the $x$ axis at $x= \pm d / 2$, use the same mass $\tilde{M}=1$ for both black holes, and set the boost velocity to $\left(0, \pm d \Omega_{0} / 2,0\right)$. The conformal spins are always equal and are aligned with the orbital angular momentum of the holes.

In contrast to the CFMS data, there are now two parameters that influence the black holes' spins: (i) the rotation parameter $\Omega_{r}$ in Eq. (40) and (ii) the conformal spin $\tilde{S}$. For concreteness, we choose to construct data for four different values of the conformal spin: $\tilde{S} / \tilde{M}^{2}=0,0.5,0.93$, and 0.99. For each choice, we construct a family of initial-data sets for different values of $\Omega_{r}$, which we label as SKS-0.0, SKS-0.5, SKS-0.93, and SKS-0.99, respectively.

Other choices that went into the construction of the SKS initial-data sets are as follows:

(i) The excision boundaries are chosen to be the coordinate locations of the horizons of the individual Kerr-Schild metrics; i.e., they are surfaces of constant Kerr radius

$$
r_{\mathrm{exc}}^{\mathrm{Kerr}}=\tilde{r}_{+}:=\tilde{M}+\sqrt{\tilde{M}^{2}-\tilde{S}^{2}}
$$

length-contracted by the Lorentz factor appropriate for the boost velocity of each black hole. This length contraction accounts for the tangential motion of the hole but neglects the much smaller radial motion.

(ii) When superposing the individual Kerr-Schild metrics, we use a damping length scale $w=10 r_{\mathrm{exc}}^{\mathrm{Ker}}[\mathrm{cf}$. Eqs. (45) and (46)], except for the SKS-0.99 family, which uses $w=d / 3$.

(iii) The orbital frequency $\Omega_{0}$ and radial expansion $\dot{a}_{0}$ are held fixed along each family. We expect that our choices for $\Omega_{0}$ and $\dot{a}_{0}$ will lead to bounded, fairly circular orbits, since 


$$
\frac{\left|E_{\mathrm{ADM}}-M_{K}\right|}{E_{\mathrm{ADM}}} \lesssim 3 \% \text {. }
$$

In Sec. V B we reduce the orbital eccentricity for one data set in the family SKS- 0.93 .

We again solve the XCTS equations using the spectral elliptic solver of Ref. [62]; the families of SKS initial-data sets that we construct are summarized in Table I. The elliptic solver needs some initial guess for the variables to be solved for; we superpose the respective single-blackhole Kerr-Schild quantities, i.e.,

$$
\begin{aligned}
\psi & =1, \\
\alpha \psi & =1+\sum_{a=1}^{n} e^{-r_{a}^{2} / w_{a}^{2}}\left(\alpha_{a}-1\right), \\
\beta^{i} & =\sum_{a=1}^{n} e^{-r_{a}^{2} / w_{a}^{2}} \beta_{a}^{i},
\end{aligned}
$$

where $n=2$ and $\alpha_{a}$ and $\beta_{a}^{i}$ are the lapse and shift, respectively, corresponding to the boosted, spinning KerrSchild metrics $g_{i j}^{a}$ used in the conformal metric $\tilde{g}_{i j}$. Convergence of the elliptic solver and spin is demonstrated in Fig. 10 by showing the decreasing constraint violation ${ }^{8}$ and differences in spin with increasing resolution.

We now turn our attention to the physical properties of the SKS initial-data sets. Figure 11 shows the horizon mass $M$ and the dimensionless spin $\chi$ of either black hole for the four families of SKS initial data. As expected, we find that generally the spin $\chi$ increases with increasing $\Omega_{r}$. For each of the SKS families, we find that the elliptic solver fails to converge for sufficiently large $\Omega_{r}$. We suspect that the SKS families exhibit a turning point, similar to the CFMS-single and binary-black-hole initial data shown in Figs. 7 and 9. If this is the case, Fig. 11 only shows the lower branch of each family, and an additional branch of solutions will be present. Because we are satisfied with the spin magnitudes that are possible along the lower branch, we do not attempt to find the upper branch here.

In contrast to the CFMS data sets (where the lower branch only allowed spins as large as $\chi \lesssim 0.85$ ), the SKS initial data allow spins that are quite close to unity. For the different SKS families, we are able to construct initial data with spins as large as

(i) $\chi \approx 0.95$ for SKS- 0 ,

(ii) $\chi \approx 0.985$ for SKS- 0.5 ,

(iii) $\chi \approx 0.998$ for SKS-0.93,

(iv) $\chi \approx 0.9997$ for SKS-0.99.

These spins are far closer to extremal than possible with Bowen-York initial data $[\chi \lesssim 0.984$ (Fig. 2)] or conformally flat, maximally sliced XCTS initial data $[\chi \lesssim 0.85$

\footnotetext{
${ }^{8}$ The constraint violation is $\sqrt{\|\mathcal{C}\|_{L 2}^{2}+\|\mathcal{C}\|_{L 2}^{i}\|\mathcal{C}\|_{L 2}^{j} \delta_{i j}}$, where $\mathcal{C}$ and $\mathcal{C}^{i}$ are the residuals of Eqs. (10) and (11) and the L2 norm is given by Eq. (73).
}

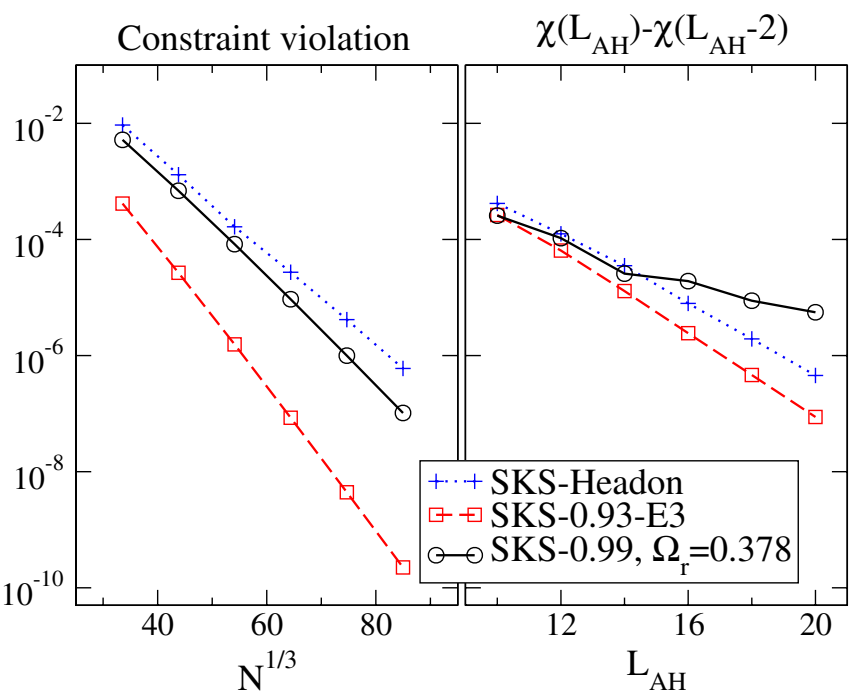

FIG. 10 (color online). Convergence of the spectral elliptic solver. Left panel: The residual constraint violation as a function of the total number of grid points $N$ when running the elliptic solver at several different resolutions. Right panel: Convergence of the black-hole dimensionless spin $\chi$ [Eq. (1)] with increasing resolution $L_{\mathrm{AH}}$ of the apparent horizon finder, applied to the highest-resolution initial-data set of the left panel. The three curves in each panel represent three different initial-data sets: one from the family SKS-0.99, as well as the two initial-data sets that are evolved in Sec. V.

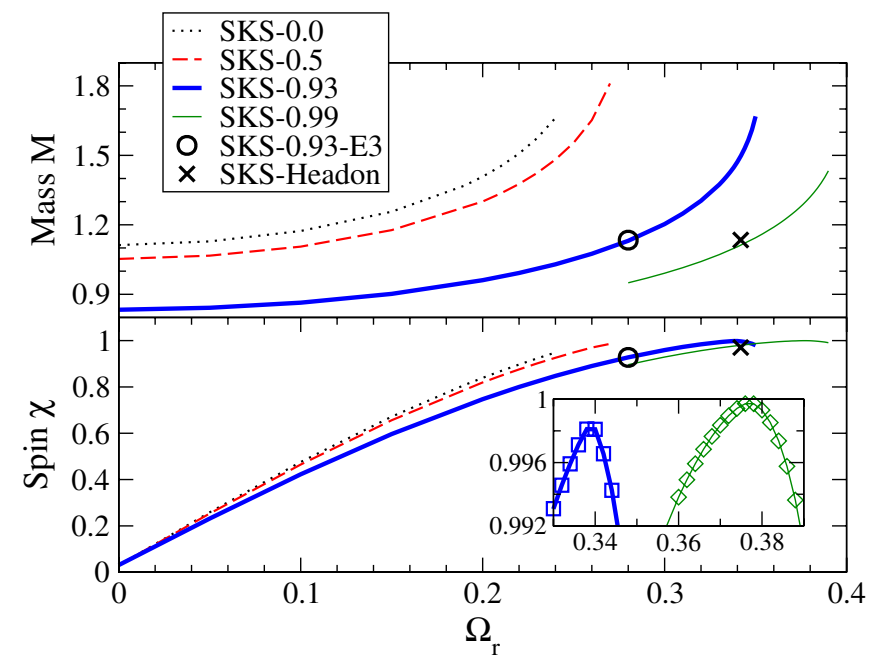

FIG. 11 (color online). The mass $M$ (upper panel) and dimensionless spin $\chi$ (lower panel) of one of the holes for superposed Kerr-Schild, binary-black-hole initial-data sets with spins aligned with the orbital angular momentum. The mass and spin are plotted against $\Omega_{r}$ [Eq. (40)] for four different choices of the conformal spin: $\tilde{S}=0,0.5,0.93$, and 0.99 . Also shown are the data sets SKS-0.93-E3-identical to the $\Omega_{r}=0.28 \tilde{M}, \tilde{S}=$ $0.93 \tilde{M}^{2}$ data set on the solid curve but with lower eccentricityand SKS-Headon; both sets are evolved in Sec. V. The inset in the lower panel shows a close-up of the spins as they approach unity, with symbols denoting the individual data sets. 
or $\$ 0.99$ along the lower and upper branch, respectively (Fig. 7)].

We note that the spins in the SKS binary-black-hole initial-data families are only weakly dependent on the orbital parameters $\Omega_{0}$ and $\dot{a}_{0}$. This can be seen from the individual data point labeled SKS-0.93-E3 shown in Fig. 11. This data set uses different values for $\Omega_{0}$ and $\dot{a}_{0}$ but is nevertheless close to the family SKS-0.93. The initial-data sets SKS-0.93-E3 and SKS-Headon will be discussed in detail in Sec. V.

The inset of Fig. 11 highlights a remarkable feature of the SKS-0.93 and SKS-0.99 families: With increasing $\Omega_{r}$, the spin initially increases but eventually decreases. Figure 12 investigates this behavior in more detail, where this effect is more clearly visible in the lower two panels: Both the spin $\chi$ and the extremality parameter $\zeta$ of the apparent horizon change direction and begin to decrease. For $\Omega_{r}$ smaller than this critical value, the apparent horizon finder always converges onto the excision surfaces, which by virtue of the boundary condition Eq. (39) are guaranteed to be marginally trapped surfaces. As $\Omega_{r}$ is increased through the critical value (at which $\chi$ and $\zeta$ change direction), a second marginally trapped surface (solid line) splits

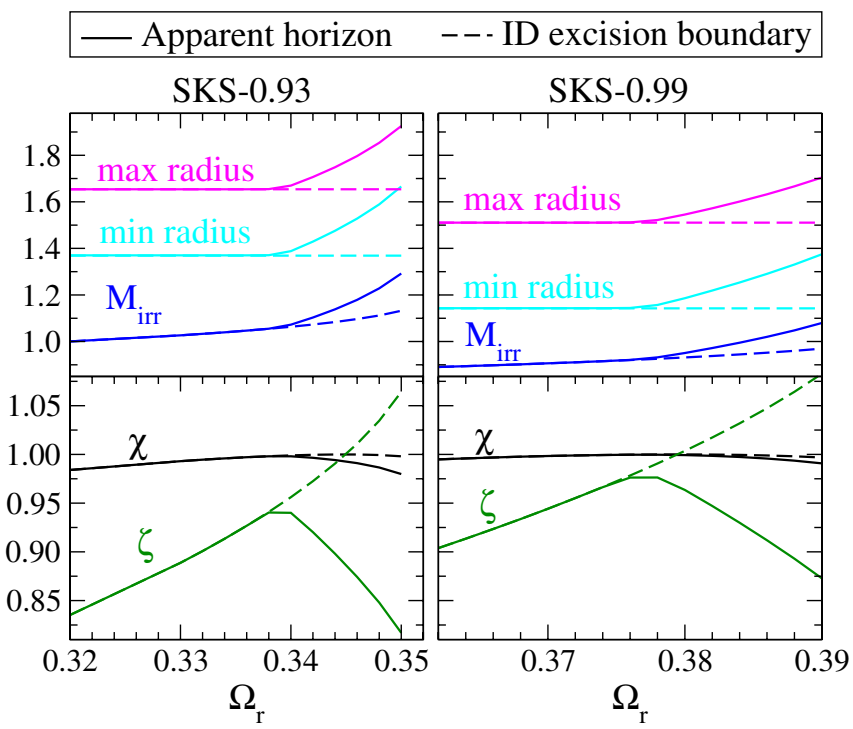

FIG. 12 (color online). The irreducible mass $M_{\text {irr }}$ and Euclidean coordinate radius $r$ (upper panels) and dimensionless spin $\chi:=S / M^{2}$ and spin-extremality parameter $\zeta:=S /\left(2 M_{\mathrm{irr}}^{2}\right)$ (lower panels) for one of the black holes in the SKS-0.93 (left) and SKS-0.99 (right) initial-data-set families. These quantities are computed on two surfaces: (i) the apparent horizon (solid lines) and (ii) the excision boundary of the initial data (dashed lines). Because we enforce that the excision surface is a marginally trapped surface, typically the apparent horizon and excision boundary coincide. However, if $\Omega_{r}$ is increased beyond the values where $\chi$ approaches unity, the apparent horizon lies outside of the excision surface. The excision surface can obtain superextremal spins $(\zeta>1)$ but only when it is enclosed by a subextremal horizon. off from the excision surface (dashed line) and moves continuously outward. This can be seen in the upper panels of Fig. 12, which plot the minimal and maximal coordinate radius and the irreducible mass of both the excision surface and the outermost marginally trapped surface, which is by definition the apparent horizon.

But what about the excision surface? The boundary condition Eq. (39) forces the excision surface to be a marginally trapped surface, independent of the value of $\Omega_{r}$. For sufficiently large $\Omega_{r}$, however, the excision surface is surrounded by a larger marginally trapped surface and thus is not the apparent horizon. The dashed lines in Fig. 12 present data for the excision surface. These lines continue smoothly across the point where the second marginally trapped surface forms. The extremality parameter $\zeta$ for the excision surface continues to increase and eventually becomes larger than unity; the excision surface can then be thought of as having a superextremal spin. However, for the outer marginally trapped surface-the true apparent horizon - the extremality parameter always satisfies $\zeta<$ 1 . The irreducible mass $M_{\text {irr }}$ of this surface increases faster than the spin, and therefore $\zeta=S /\left(2 M_{\mathrm{irr}}^{2}\right)$ decreases with increasing $\Omega_{r}$.

One might interpret these results as support of the cosmic censorship conjecture. The XCTS boundary conditions (39) and (40) control the location and the spin of the excision surface. By appropriate choices for the shift boundary condition (40), we can force the excision surface to become superextremal. However, before this can happen, a new horizon appears, surrounding the excision surface and hiding it from "our" asymptotically flat end of the spacetime. The newly formed outer horizon always remains subextremal.

\section{Suitability for evolutions}

In the previous sections, we have constructed a wide variety of binary-black-hole initial-data sets. To get some indication about how suitable these are for evolutions, we consider the initial time derivatives of these data sets $\partial_{t} g_{i j}$ and $\partial_{t} K_{i j}$. Recall that solutions of the XCTS equations give a preferred initial lapse and shift for the evolution of the initial data; hence, the time derivatives $\partial_{t} g_{i j}$ and $\partial_{t} K_{i j}$ can be computed by simply substituting the initial data into the ADM evolution equations. We expect initial data with smaller time derivatives to be closer to quasiequilibrium and to have less initial spurious radiation.

Figure 13 presents the L2 norms of the time derivatives $\left\|\partial_{t} g_{i j}\right\|_{L 2}$ and $\left\|\partial_{t} K_{i j}\right\|_{L 2}$ where the L2 norm of a tensor $T_{i j k \cdots} \ldots(x)$ evaluated at $N$ grid points $x_{i}$ is defined as

$$
\left\|T_{i j k \cdots}\right\|_{L 2}:=\sqrt{\frac{1}{N} \sum_{i=0}^{N} \bar{T}^{2}\left(x_{i}\right)},
$$

where 


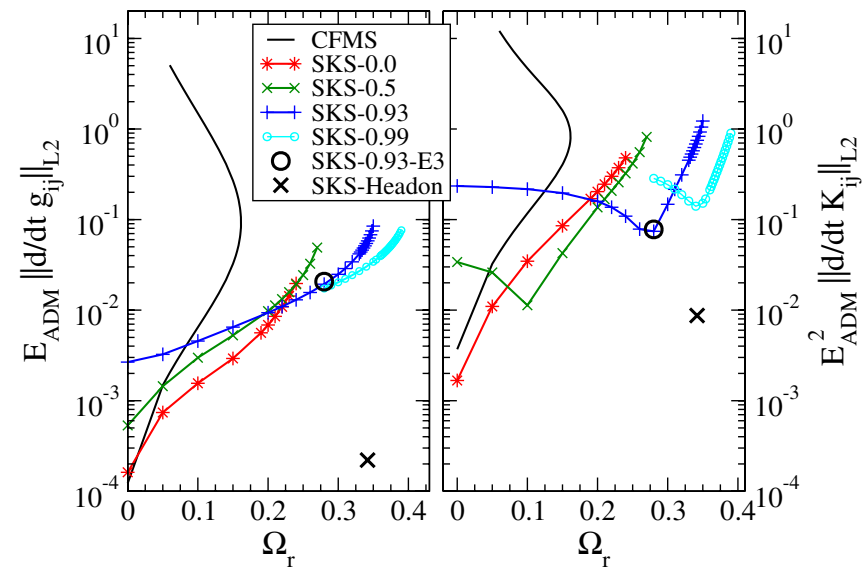

FIG. 13 (color online). The time derivatives of the metric (left panel) and extrinsic curvature (right panel). In the SKS data sets, $\left\|\partial_{t} K_{i j}\right\|_{L 2}$ has minima near values of $\Omega_{r}$ for which the dimensionless spin $\chi$ is approximately equal to the spin $\tilde{S}$ of the conformal metric (cf. Fig. 11). On the upper branch of the CFMS excision data, where the spin is $\chi>0.83$ (Fig. 9), the time derivatives become much larger than the SKS time derivatives. The data sets SKS-0.93-E3 (with $\chi \approx \tilde{S}=0.93$ ) and SKSHeadon (with $\chi \approx \tilde{S}=0.97$ ) are evolved in Secs. V C and VD; the time derivatives are significantly lower for the set SKSHeadon because of the larger coordinate separation of the holes $(d=100$ vs $d=32)$.

$$
\bar{T}:=\sqrt{T_{i j k \ldots} T_{i^{\prime} j^{\prime} k^{\prime} \ldots} \delta^{i i^{\prime}} \delta^{j j^{\prime}} \delta^{k k^{\prime}} \ldots}
$$

Figure 13 shows that generally $\partial_{t} K_{i j}$ is larger than $\partial_{t} g_{i j}$. This has also been found in previous work, e.g., [63], and is not surprising, because the XCTS formalism allows some control over the time derivative of the metric through the free data $\tilde{u}_{i j}=\partial_{t} \tilde{g}_{i j}$, whereas there is less control of $\partial_{t} K_{i j}$. We note that for CFMS data, the time derivatives are larger and grow more rapidly with $\chi$ than for SKS data; in particular, the time derivatives on the upper branch are $\sim 10$ times larger than for SKS-initial data, suggesting that these data are much farther from equilibrium.

In the SKS case, the time derivatives of $K_{i j}$ have local minima at particular values of $\Omega_{r}$; comparison with Fig. 11 gives spins $\chi$ at these minima of $\left\|\partial_{t} K_{i j}\right\|_{L 2}$ as follows:

(i) SKS-0.5: $\Omega_{r} \approx 0.1, \chi \approx 0.45$;

(ii) SKS-0.93: $\Omega_{r} \approx 0.28, \chi \approx 0.93$;

(iii) SKS-0.99: $\Omega_{r} \approx 0.34, \chi \approx 0.98$.

Note that these minima occur at values of $\Omega_{r}$ such that $\chi \approx$ $\tilde{S} / \tilde{M}^{2}$; that is, transients in the initial data and presumably the spurious radiation are minimized when the conformal spin and AKV spin are consistent. For this reason, we conclude that SKS initial data with $\chi \approx \tilde{S} / \tilde{M}^{2}$ is preferable; this is the type of initial data we will evolve in the next section.

Also note that minimizing the spurious radiation has purely numerical advantages: The spurious radiation typi- cally has finer structure (and thus requires higher resolution) than the physical radiation. If such radiation is minimized, the numerical evolutions may require less resolution and will be more efficient. Conformally curved initial data has been found to reduce the amount of spurious radiation in Refs. [39,64].

\section{EXPLORATORY EVOLUTIONS OF SKS INITIAL DATA}

So far, we have confined our discussion to black-hole spins in the initial data. In this section, we compare the initial spin to the value to which the spin relaxes after the initial burst of spurious radiation, when the holes have settled down. Recall, for instance, that for Bowen-York puncture initial data with spins close to the maximal possible value $[\chi(t=0) \approx 0.98]$, the spins quickly relax by about $\Delta \chi \approx 0.05$ to a maximal possible relaxed value of $\chi\left(t_{\text {relax }}\right) \approx 0.93$ (cf. [25]). While the SKS data presented in Sec. IV B can achieve larger initial spins $[\chi(t=0)=$ 0.9997] than conformally flat puncture data, only evolutions can determine $\Delta \chi$ and $\chi\left(t_{\text {relax }}\right)$.

Therefore, in this section we perform brief, exploratory evolutions of some SKS initial-data sets to determine $\Delta \chi$ for those data sets. ${ }^{9}$ Besides determination of $\chi\left(t_{\text {relax }}\right)$, these evolutions will also allow us to demonstrate that the technique of eccentricity reduction developed in Ref. [49] is applicable to SKS initial data as well as to compare the spin measures defined in Appendixes A and B. The focus here lies on initial data, and we evolve only long enough for our purposes. Longer simulations that continue through merger and ringdown are the subject of ongoing research.

This section is organized as follows. In Sec. VA, we summarize the evolution code that we will use. In Sec. V B, we perform eccentricity reduction on one of the data sets in the SKS-0.93 family, which corresponds to an orbiting binary black hole with equal masses and equal spins (of magnitude $\chi \approx 0.93$ ) aligned with the orbital angular momentum. Then, in Sec. VC, we evolve the resulting loweccentricity data set (labeled SKS-0.93-E3). Finally, in Sec. VD, we evolve a head-on plunge of SKS initial data (labeled SKS-Headon) representing two widely separated black holes with initial spins of magnitude $\chi=0.970$ and direction normal to the equatorial plane.

\section{A. Description of evolution code}

The initial data are evolved using the Caltech-Cornell pseudospectral evolution code SpEC [65]. The details of the evolution methods, equations, and boundary conditions that we use are the same as those described in Ref. [66]. The singularities are excised, with the excision surfaces

\footnotetext{
${ }^{9}$ Note that there is no universal value of $\Delta \chi$-it will differ for different initial-data sets, even within the same family of initial data.
} 
chosen to lie slightly inside the black-hole horizons. Note that whereas Ref. [66] excises coordinate spheres inside the black holes' apparent horizons, here we use Lorentzcontracted ellipsoidal excision boundaries which are adapted to the shape of the initial apparent horizons.

The highest-resolution initial-data set (with $N \approx 85^{3}$ grid points) is interpolated onto evolution grids labeled $\mathrm{N} 1, \mathrm{~N} 2$, and N3 with approximately $61^{3}, 67^{3}$, and $74^{3}$ grid points, respectively. The outer boundary is at a coordinate radius of $r=32 d$ for the orbiting simulation discussed in Secs. V B and V C and at $r=14 d$ for the head-on simulations discussed in Sec. V D. This translates to about $r=450 E_{\mathrm{ADM}}$ and $r=620 E_{\mathrm{ADM}}$ for the orbiting and headon simulations, respectively. As in earlier simulations $[49,65,66]$, a small region of the evolution grid lies inside the horizon and is not covered by the initial-data grid; we extrapolate $\psi, \alpha \psi$, and $\beta^{i}$ into this region and then compute $g_{i j}$ and $K_{i j}$.

\section{B. Eccentricity removal for orbiting SKS binaries}

We obtain initial data with small orbital eccentricity using the iterative method of Ref. [49], as refined in Ref. [66], applied here for the first time to binary-blackhole data with rapid spin. In this method, the choice of $\Omega_{0}$ and $\dot{a}_{0}$ for the next iteration are made so that if the orbit were Newtonian, the eccentricity would vanish. For the non-Newtonian orbit here, successive iterations succeed in reducing the orbital eccentricity.

This procedure is based on the proper separation $s$ between the apparent horizons, measured along a coordinate line connecting the geometric centers of the apparent horizons. The time derivative $d s / d t$ is fitted to a fiveparameter curve that, together with the initial proper separation $s(t=0)$, is used to define the eccentricity $e$ and to define improved values for $\Omega_{0}$ and $\dot{a}_{0}$. Specifically,

$$
\begin{aligned}
\frac{d s}{d t} & :=A_{0}+A_{1} t+B \cos (\omega t+\varphi), \\
e & :=\frac{B}{\omega s(t=0)}, \\
\Omega_{0, \text { new }} & :=\Omega_{0}+\frac{B \sin \phi}{2 s(t=0)}, \\
\dot{a}_{0, \text { new }} & :=\dot{a}_{0}-\frac{B \cos \phi}{s(t=0)} .
\end{aligned}
$$

Heuristically, the eccentricity is embodied by the oscillating part of $d s / d t$.

Figure 14 illustrates the eccentricity reduction for one of the data sets in family SKS-0.93. Plotted are the proper separation $s$ and its derivative $d s / d t$ for evolutions of several initial-data sets (summarized in Table I):

(i) set SKS-0.93-E0, which is identical to the set in family SKS-0.93 with $\Omega_{r}=0.28$ (Fig. 11);

(ii) set SKS-0.93-E1, which is the same as SKS-0.93-E0 except that the orbital frequency $\Omega_{0}$ is manually

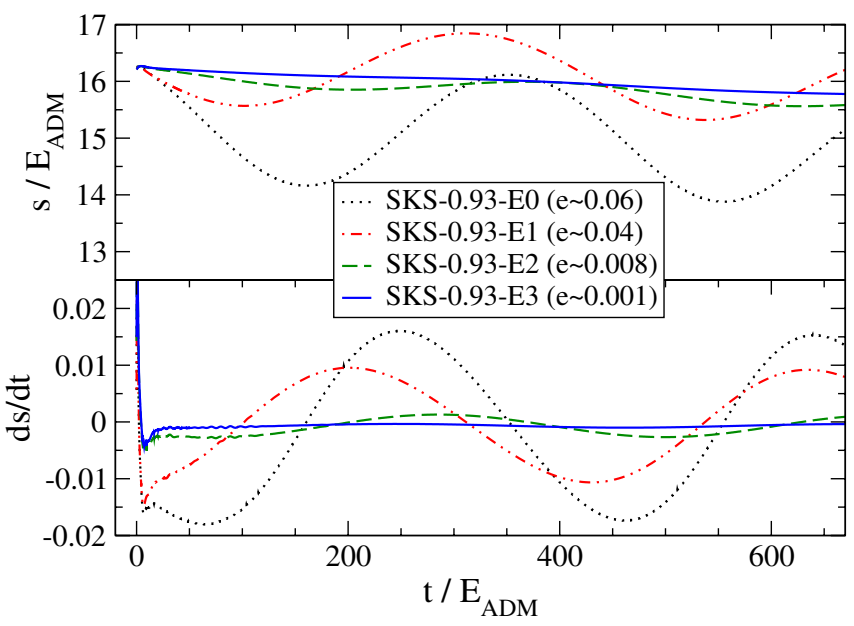

FIG. 14 (color online). Eccentricity reduction for evolutions of superposed Kerr-Schild binary-black-hole initial data. The proper separation $s$ (upper panel) and its time derivative $d s / d t$ (lower panel) are plotted for initial-data sets SKS-0.93-E0, -E1, -E2, and -E3, which have successively smaller eccentricities $e$. All evolutions are performed at resolution N1.

adjusted to lower the orbital eccentricity somewhat; and

(iii) sets SKS-0.93-E2 and SKS-0.93-E3, which are successive iterations (starting from set SKS-0.93E1) of the eccentricity-reduction scheme Eqs. (75).

The ad hoc adjustment of $\Omega_{0}$ was somewhat effective, reducing $e$ by about $50 \%$. The subsequent iterations using Eqs. (75) reduced $e$ by factors of about 5 and 8, respectively. Surprisingly, the lowest eccentricity, corresponding to a smooth inspiral trajectory, is obtained with a positive $\dot{a}_{0}=3.332 \times 10^{-4}$. This is not due to insufficient resolution; for SKS-0.93-E3, we have verified that we obtain the same eccentricity $e \sim 0.001$ for all three numerical resolutions N1, N2, and N3.

Note that we choose to stop the evolutions at about $t=$ $670 E_{\mathrm{ADM}}$, which corresponds to about 1.9 orbits; this is sufficient for reducing the eccentricity and for measuring $\Delta \chi$. In the next subsection, we discuss the evolution of the low-eccentricity set SKS-0.93-E3 in detail, focusing on the relaxation of the spin $\chi$.

\section{Low-eccentricity inspiral with $\boldsymbol{\chi} \approx \mathbf{0 . 9 3}$}

We evolved the data set SKS-0.93-E3 at three different numerical resolutions for a duration of about $670 E_{\mathrm{ADM}}$, corresponding to about 1.9 orbits. From post-Newtonian theory [67], we estimate that this simulation would proceed through about 20 orbits to merger.

Figure 15 presents a convergence test for this run. The lower panel of Fig. 15 shows the normalized constraint violation [see Eq. (71) of Ref. [68] for the precise definition]. While the constraints are small, the convergence seems poor until $t \approx 500 E_{\mathrm{ADM}}$. For this time period the 
BINARY-BLACK-HOLE INITIAL DATA WITH NEARLY- ...

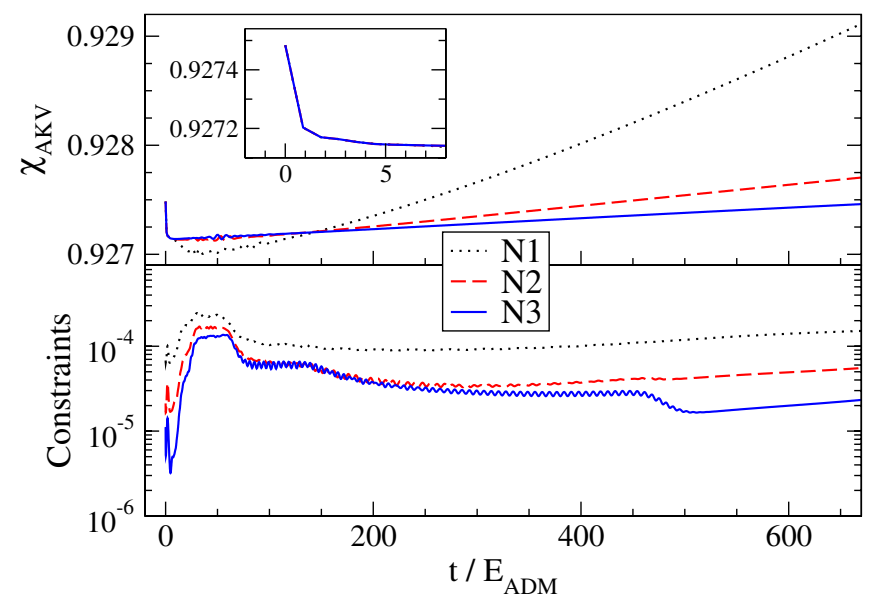

FIG. 15 (color online). Convergence test of the evolution of the initial-data set SKS-0.93-E3. Shown are evolutions on three different resolutions N1, N2, and N3, with N3 being the highest resolution. The top panel shows the AKV spin of one of the holes as a function of time, with the top inset showing the spin's initial relaxation; the bottom panel shows the constraint violation as a function of time.

constraint violations at high resolution N3 are dominated by the outgoing pulse of spurious radiation-i.e., far away from the black holes-which we have not attempted to adequately resolve. At $t \approx 500 E_{\mathrm{ADM}}$, the pulse of spurious radiation leaves the computational domain through the outer boundary; afterwards, the constraints decrease exponentially with increasing resolution, as expected.

The upper panel of Fig. 15 shows the AKV spin $\chi_{\mathrm{AKV}}=$ $S / M^{2}$ for the three runs with different resolutions N1, N2, and N3. Based on the difference between N2 and N3, the spin of the evolution $\mathrm{N} 3$ should be accurate to a few parts in $10^{4}$. For the time interval $5<t / E_{\mathrm{ADM}}<670$, the measured spin on resolution N3 is consistent with begin constant within its estimated accuracy. Very early in the simulation, $t<5 E_{\mathrm{ADM}}$, the spin $\chi$ changes convergently resolved from its initial value $\chi(t=0)=0.92748$ to a relaxed value $\chi\left(t_{\text {relax }}\right)=0.92714$ (see inset of Fig. 15). Therefore, for SKS-0.93-E3, we find $\Delta \chi=0.00034$.

Contrast this result with the evolution of a binary-blackhole puncture initial-data set with large spins, which is reported in Ref. [25]: For that particular evolution, $\chi(t=$ $0)=0.967, \chi\left(t_{\text {relax }}\right)=0.924$, i.e., $\Delta \chi=0.043$, more than a factor 100 larger than for the evolution of SKS-0.93-E3 reported here. This comparison is somewhat biased against the puncture evolution in [25], which starts at a smaller separation possibly resulting in larger initial transients. However, even in the limit that the black holes are infinitely separated (i.e., in the single-black-hole limit), the spins in Bowen-York puncture data relax to values near $\varepsilon_{J}=J_{\mathrm{ADM}} / E_{\mathrm{ADM}}^{2}$; to achieve a final spin of $\chi\left(t_{\text {relax }}\right) \approx$ 0.93 , the initial spin of Bowen-York data must be $\chi(t=$ $0) \approx 0.98$ (cf. Fig. 2 of Ref. [25]). We conclude that the
PHYSICAL REVIEW D 78, 084017 (2008)

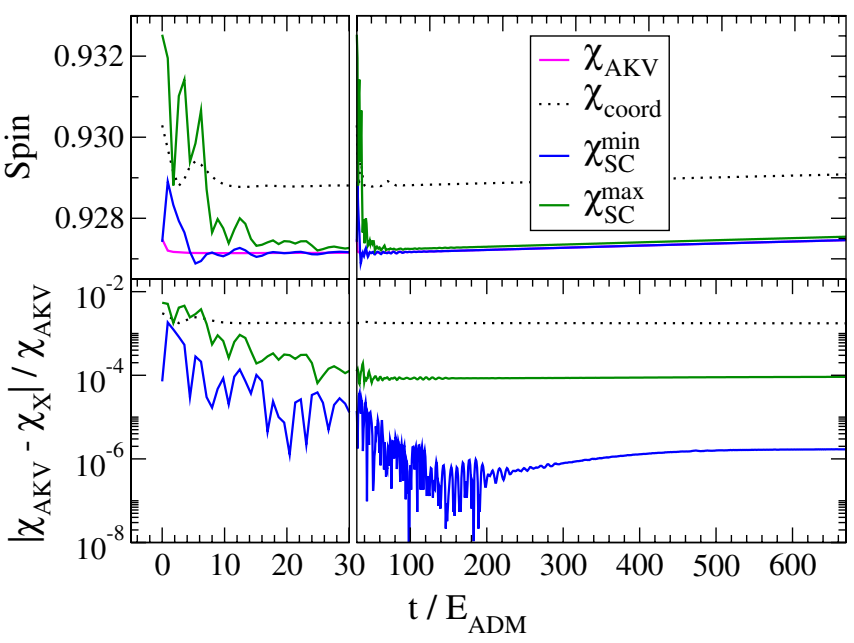

FIG. 16 (color online). A comparison of different definitions of the spin. The top panel shows the spin as a function of time for several different measures of the spin; the bottom panel shows the fractional difference between $\chi_{\mathrm{AKV}}$ and alternative spin definitions. Note that for $t<30 E_{\mathrm{ADM}}$, the time axis has a different scaling to make the initial transients visible.

spin relaxes by a much smaller amount in the SKS case than in Bowen-York puncture or inversion-symmetric data.

Figure 15 and the discussion in the previous paragraph only address the behavior of the AKV spin, where the approximate Killing vectors are computed from the minimization problem [cf. Eq. (A10)]. We now compare the different spin definitions we present in Appendixes A and B. Figure 16 compares these different definitions of the black-hole spin for the N3 evolution of initial-data set SKS-0.93-E3. Shown are the AKV spin of one hole in the binary, the scalar-curvature (SC) spins $\chi_{\mathrm{SC}}^{\min }$ and $\chi_{\mathrm{SC}}^{\max }$ of Appendix B [Eqs. (B2a) and (B2b)], and also the spin obtained by using Eq. (A1) with a coordinate rotation vector instead of an approximate Killing vector (which we call the "coordinate spin" here). After the holes have relaxed, the SC spins track the AKV spin more closely than does the coordinate spin. However, during very early times, as the holes are relaxing and the horizon shape is very distorted, the SC spins show much larger variations. Consequently, the SC spin is a poorer measure of the spin at early times than even the coordinate spin.

\section{Head-on plunge with $\chi \approx 0.97$}

In the previous subsection, we have seen that, for SKS binary-black-hole initial data with $\chi=0.93$, the initial spins change by only a few parts in $10^{4}$. A spin $\chi \approx 0.93$ is roughly the largest possible equilibrium spin that is obtainable using standard conformally flat, Bowen-York puncture data (cf. the discussion at the beginning of Sec. V). We now begin to explore binary-black-hole simulations with spin magnitudes that are not obtainable with Bowen-York initial-data methods. 
We construct and evolve SKS binary-black-hole data for a head-on plunge of two equal-mass black holes with spins of equal magnitude $\chi=0.97$ and with the spins orthogonal to the line connecting the black holes. This data set, labeled SKS-Headon, is summarized in Table I and was briefly discussed in Sec. IV B; cf. Figs. 10, 11, and 13. As for the orbiting evolution SKS-0.93-E3, we adjust the rotation parameter $\Omega_{r}$ so that conformal spin $\tilde{S} / \tilde{M}^{2}$ and AKV spin $\chi$ are approximately equal. Starting such a simulation at close separation results in rapid coordinate motion of the apparent horizons during the first few $E_{\mathrm{ADM}}$ of the evolution. These motions are currently difficult to track with our excision code; therefore, we begin at a larger separation $d$ than we used in the nearly circular data sets described previously.

Figure 17 presents a convergence test of the constraints (lower panel) and the AKV spin $\chi_{\mathrm{AKV}}$ (upper panel) during the subsequent evolution. Again, we are interested in the initial relaxation of the spins; therefore, we choose to stop evolution at $t \approx 120 E_{\mathrm{ADM}}$. During this time, the black-hole proper separation decreased from $s(t=0)=47.6 E_{\mathrm{ADM}}$ to $s(t=120)=44.1 E_{\mathrm{ADM}}$.

During the first $\sim 10 E_{\mathrm{ADM}}, \chi_{\mathrm{AKV}}$ shows (a numerically resolved) decrease of about $3 \times 10^{-5}$; this change arises due to initial transients as the black holes and the full geometry of the spacetime relax into an equilibrium configuration. Subsequently, the spin remains constant to within about $10^{-4}$, where these variations are dominated by numerical truncation error.

Figure 18 compares our various spin measures for the head-on simulation. Interestingly, the spin $\chi_{\text {coord }}$ computed from coordinate rotation vectors agrees much better with $\chi_{\mathrm{AKV}}$ than for the SKS-0.93-E3 evolution, perhaps because the black holes here are initially at rest. The SC spins $\chi_{\mathrm{SC}}^{\mathrm{min}}$

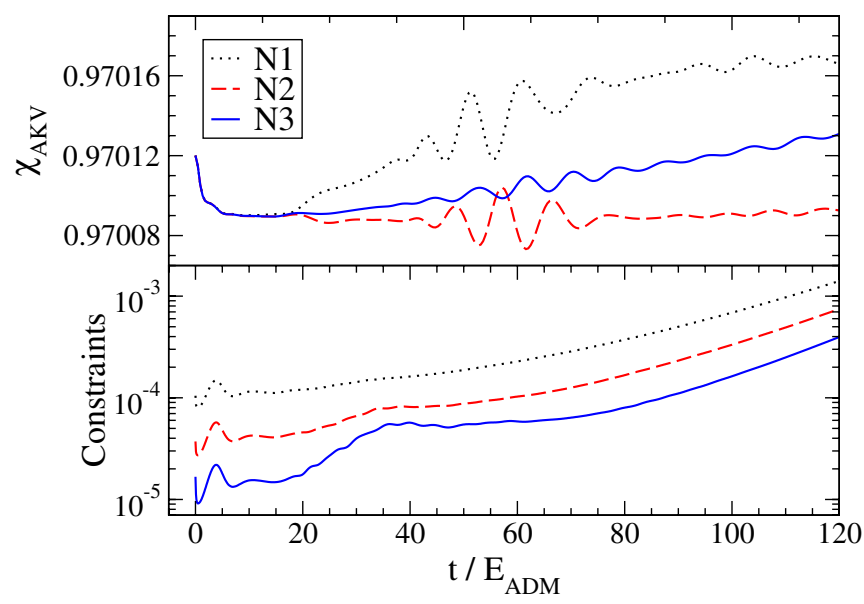

FIG. 17 (color online). Convergence test of the head-on evolution SKS-Headon. Shown are evolutions at three different resolutions $\mathrm{N} 1, \mathrm{~N} 2$, and $\mathrm{N} 3$, with $\mathrm{N} 3$ being the highest resolution. The top panel shows the AKV spin of one of the holes as a function of time; the bottom panel shows the constraint violations as a function of time.

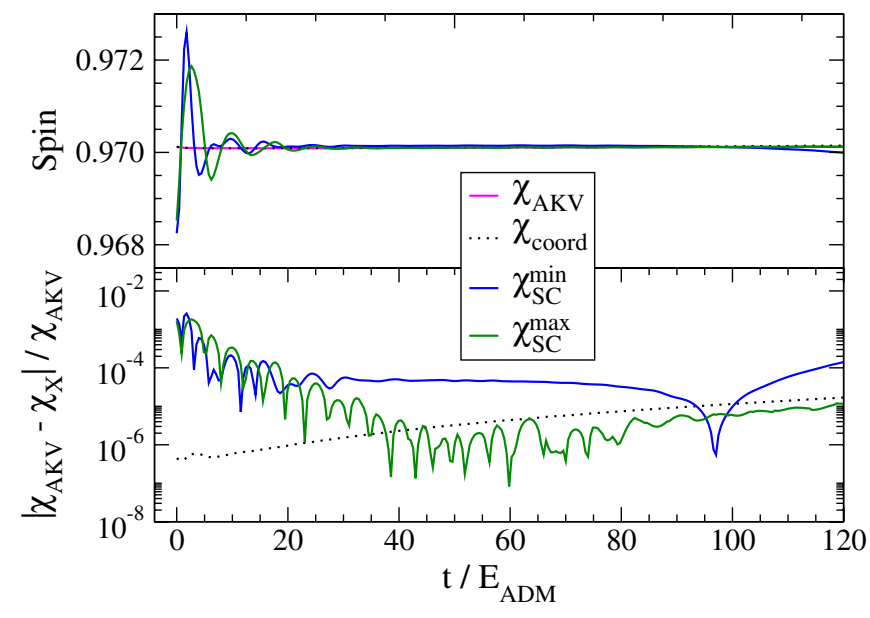

FIG. 18 (color online). A comparison of various measures of the spin for the head-on evolution of data set SKS-Headon, which is a plunge of two equal-mass black holes with parallel spins of magnitude $\chi_{\mathrm{AKV}}=S / M^{2}=0.970$ pointed normal to the equatorial plane. The top panel shows various measures of the spin as a function of time, and the bottom panel shows the fractional difference between the AKV spin $\chi_{\mathrm{AKV}}$ and alternative spin definitions.

and $\chi_{\mathrm{SC}}^{\max }$, derived from the scalar curvature of the apparent horizon [Eqs. (B2a) and (B2b)], show some oscillations at early times; after the initial relaxation, the $\mathrm{SC}$ spin agrees with the AKV spin to about 1 part in $10^{4}$.

\section{DISCUSSION}

\section{A. Maximal possible spin}

In this paper, we have examined a variety of methods for constructing black-hole initial data with a particular emphasis on the ability to construct black holes with nearly extremal spins. These are spins for which the dimensionless spin $\chi=S / M^{2}$ and spin-extremality parameter $\zeta=$ $S /\left(2 M_{\text {irr }}^{2}\right)$ are close to unity.

When discussing black-hole spin, one needs to distinguish between the initial black-hole spin and the relaxed spin of the holes after they have settled down. Using conformally flat BY data (both puncture data or inversion-symmetric data) for single black holes, the largest obtainable spins are $\chi \approx 0.984, \zeta \approx 0.833$ (cf. Ref. [61] and Fig. 2). With CFMS, QE-XCTS data, we are able to obtain initial spins as large as $\chi \approx 0.99, \zeta \approx$ 0.87 for single black holes (Fig. 7). The limitations of BY puncture data and CFMS QE-XCTS data are already present when constructing highly spinning single black holes; therefore, we expect the methods to be able to construct binary-black-hole data with similar spins as for single holes-i.e., up to about 0.98 . Construction of CFMS QE-XCTS binary-black-hole initial data confirms this conjecture (compare Fig. 9 with Fig. 7).

For SKS initial data, the situation is different. For single black holes, SKS data reduce to the analytical Kerr solu- 
tion, without any limitations on the spin magnitude. Thus limitations of SKS data will only be visible for binaryblack-hole configurations. As Secs. IV and V show, however, those limitations are quite minor. SKS data can indeed achieve initial spins that are much closer to extremality than what is possible with BY data or CFMS QE-XCTS data; we have explicitly demonstrated this by constructing SKS data for binary black holes with $\chi \approx$ $0.9997, \zeta \approx 0.98$, as can be seen from Figs. 11 and 12 .

As the black-hole spacetimes settle into equilibrium and emit spurious gravitational radiation, the initial spin $\chi$ decreases to a smaller relaxed spin $\chi\left(t_{\text {relax }}\right)$. Thus an interesting quality factor for high-spin black-hole initial data is $\Delta \chi=\chi(t=0)-\chi\left(t_{\text {relax }}\right)$ [Eq. (3)] considered as a function of the relaxed spin. The magnitude of $\Delta \chi$ is indicative of the amplitude of any initial transients, whereas the maximally achievable $\chi\left(t_{\text {relax }}\right)$ gives the largest possible spin which can be evolved with such initial data. Figure 19 presents this plot, with the circle and cross representing the two evolutions of SKS data which were described in Sec. V.

We have not evolved high-spin puncture data nor highspin CFMS-XCTS data; therefore, we do not know precisely $\Delta \chi$ for these initial data. We estimate $\Delta \chi$ for puncture data by noting that evolutions of single-hole, BY puncture data with large spins show [25] that the black-hole spin $\chi:=S / M^{2}$ relaxes approximately to the initial value of $\varepsilon_{J}:=J_{\mathrm{ADM}} / E_{\mathrm{ADM}}^{2}$. Therefore, for $\mathrm{BY}$ puncture data, we approximate

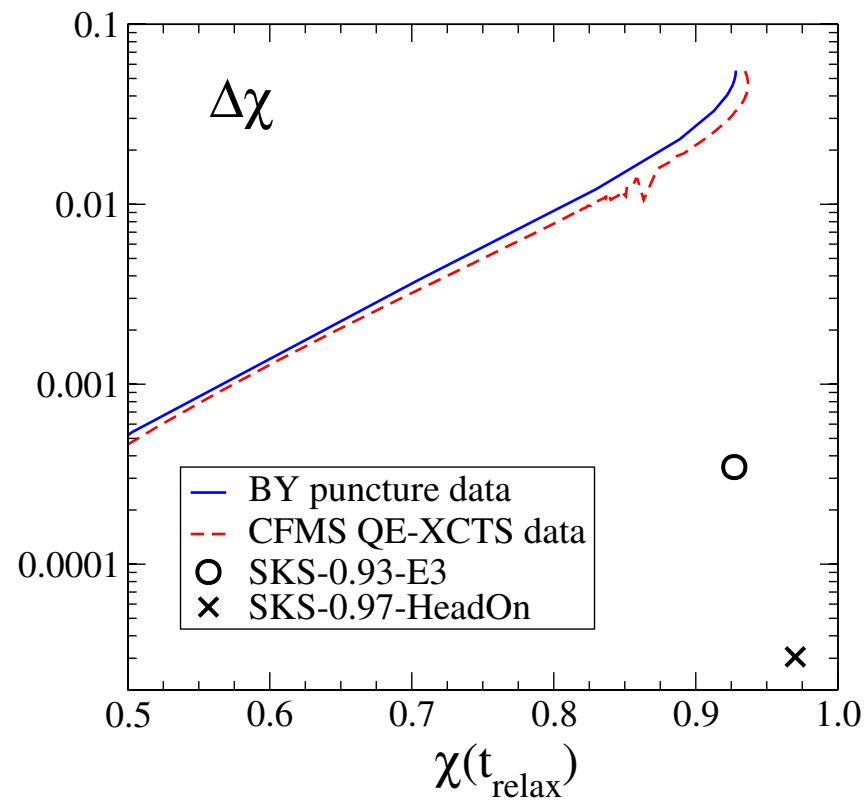

FIG. 19 (color online). The change $\Delta \chi$ in black-hole spin $\chi$ during the initial relaxation of black-hole initial data plotted as a function of the black-hole spin after relaxation. The SKS initial data constructed in this paper have smaller transients and allow for larger relaxed spins.

$$
\begin{gathered}
\Delta \chi \approx \varepsilon_{J}-\chi(t=0), \\
\chi\left(t_{\text {relax }}\right) \approx \varepsilon_{J} .
\end{gathered}
$$

This curve is plotted in Fig. 19. Because high-spin singleblack-hole, CFMS QE-XCTS initial data and BY puncture data have quite similar values of $\chi(t=0)$ and $\varepsilon_{J}$, as well as similar embedding diagrams (cf. Fig. 8), we conjecture that Eqs. (76) and (77) are also applicable to CFMS QEXCTS data. This estimate is also included in Fig. 19. We see that both types of initial data result in a $\Delta \chi$ of similar magnitude which grows rapidly with $\chi_{\text {relaxed }}$.

Perhaps the most remarkable result of Fig. 19 is the extremely small change in black-hole spin during the relaxation of SKS initial data, even at spins as large as $\chi=$ 0.97 . The small values of $\Delta \chi$ combined with the ability to construct initial data with initial spins $\chi(t=0)$ as large as 0.9997 (cf. Fig. 11) makes it highly likely that SKS initial data are capable of constructing binary black holes with relaxed spins significantly closer to unity than 0.97 . Evolutions of initial data with spins $\chi$ much closer to unity, i.e., farther into the regime that is inaccessible to conformally flat data, are a subject of our ongoing research.

In summary, the two main results of this paper are as follows:

(i) SKS initial data can make binary black holes that initially have nearly extremal spins, and

(ii) for SKS initial data, the relaxed spin is quite close to the initial spin, even when the spin is large.

\section{B. Additional results}

While working toward the main results discussed in the previous subsection, we have also established several additional interesting results. We have considered spinning, single-black-hole, puncture data which is identical to single-black-hole, spinning, inversion-symmetric data. Using this correspondence and our accurate spectral elliptic solver, we revisited the relation between black-hole spin $\chi$, specific total angular momentum of the spacetime $\varepsilon_{J}$, and the spin parameter $S$ for BY puncture data and established in Fig. 2 that both $\chi$ and $\varepsilon_{J}$ approach their limits for $S \rightarrow \infty$ as power laws; cf. Eqs (49) and (50). We have also extended the analytical analysis of Dain, Lousto, and Zlochower [25] of the throat region of high-spin puncture data toward more quantitative results, including the precise amplitudes of the conformal factor, throat circumference and throat length, as well as their scaling with spin parameter $S$ and puncture mass $m_{p}$ [Eqs. (54)-(58)]. Furthermore, Ref. [25] implicitly assumed that the throat region is approximately spherically symmetric; our Fig. 5 presents explicit evidence in support of this assumption but also shows that the throat is not precisely spherically symmetric.

We have also examined high-spin QE-XCTS initial data employing the common approximations of CFMS. With 
increasing angular frequency $\Omega_{r}$ of the horizon, we discover nonunique solutions. Thus, the nonuniqueness of the $\mathrm{XCTS}$ equations can be triggered not only by volume terms (as in [34]) but also through boundary conditions [in this case, by Eq. (40)]. Interestingly, CFMS QE-XCTS data appear to be very similar to BY puncture data, in regard to nearly extremal spins. Both data formalisms result in similar maximal values of $\chi(t=0)$ and $\varepsilon_{J}$ (Figs. 2, 7, and 19) and have embedding diagrams which develop a lengthening throat as the spin is increased (Fig. 8).

We also have found an interesting property of the horizon geometries for SKS data, which one might interpret as support of the cosmic censorship conjecture. Specifically, we find that by increasing $\Omega_{r}$ sufficiently we can in fact force the excision boundaries of the initial data to be "horizons" (i.e., marginally trapped surfaces) with superextremal spin $(\zeta>1)$. However, these superextremal surfaces are always enclosed by a larger, subextremal $(\zeta<1)$ apparent horizon.

To measure black-hole spins, we have employed and compared several different techniques to measure blackhole spin. Primarily, we use a quasilocal spin definition based on (approximate) Killing vectors [Eq. (A1)]. This formula requires the choice of an approximate Killing vector, and we have used both straightforward coordinate rotations to obtain $\chi_{\text {coord }}$ and solved Killing's equation in a least-squares sense to obtain $\chi_{\mathrm{AKV}}$ (see Appendix A for details). Furthermore, we introduced a new technique to define black-hole spin which does not require choice of an approximate Killing vector and is invariant under spatial coordinate transformations and transformations associated with the boost gauge ambiguity of the dynamical horizon formalism. This new technique is based on the extrema of the scalar curvature of the apparent horizon. Figures 16 and 18 show that all four spin measures agree to good precision, but differences are noticeable. The spin measures based on the horizon curvature exhibit more pronounced variations during the initial transients, and the quasilocal spin based on coordinate rotations is off by several tenths of a percent. The quasilocal spin based on approximate Killing vectors $\chi_{\mathrm{AKV}}$ has the smallest initial variations.

Finally, we would like to point out that a modified version of the SKS-initial data has been very successfully used to construct black-hole-neutron-star initial data [69].

\section{ACKNOWLEDGMENTS}

It is a pleasure to acknowledge useful discussions with Ivan Booth, Gregory Cook, Stephen Fairhurst, Lawrence Kidder, Lee Lindblom, Mark Scheel, Saul Teukolsky, and Kip Thorne. The numerical calculations in this paper were performed using the Spectral Einstein Code (SpEC), which was primarily developed by Lawrence Kidder, Harald Pfeiffer, and Mark Scheel. We would also like to acknowledge the anonymous referee for reminding us of an important technical caveat. Some equations in this paper were obtained using MATHEMATICA. This work was supported in part by grants from the Sherman Fairchild Foundation to Caltech and Cornell and from the Brinson Foundation to Caltech; by NSF Grants No. PHY-0652952, No. DMS0553677, and No. PHY-0652929 and NASA Grant No. NNG05GG51G at Cornell; and by NSF Grants No. PHY-0601459, No. PHY-0652995, and No. DMS0553302 and NASA Grant No. NNG05GG52G at Caltech.

\section{APPENDIX A: QUASILOCAL SPIN USING APPROXIMATE KILLING VECTORS (AKV SPIN)}

In this appendix and the one that follows, we address the task of defining the spin of a dynamical black hole, given $g_{i j}$ and $K_{i j}$. We use two different measures. The first, defined here, is a standard quasilocal angular momentum defined with approximate Killing vectors which correspond to approximate symmetries of a black hole's horizon. The second measure, defined in Appendix B, infers the spin from geometrical properties (specifically, from the intrinsic scalar curvature $\stackrel{\circ}{R}$ ) of the apparent horizon, assuming that the horizon is that of a single black hole in equilibrium, (i.e., that the horizon is that of a Kerr black hole). Note that quantities relating to the geometry of the two-dimensional apparent horizon surface $\mathcal{H}$ are denoted with a ring above them, to avoid confusion with the analogous quantities on the spatial slice $\Sigma$.

It has become standard in the numerical relativity community to compute the spin angular momentum of a black hole with the formula [70-72]

$$
S=\frac{1}{8 \pi} \oint_{\mathcal{H}} \phi^{i} s^{j} K_{i j} d A,
$$

where $s^{i}$ is the outgoing normal of $\mathcal{H}$ embedded in $\Sigma$ and $\vec{\phi}$ is an "azimuthal" vector field, tangent to $\mathcal{H}$. The azimuthal vector field $\vec{\phi}$ carries information about the "axis" about which the spin is being computed. There are, however, far more vector fields on a two-sphere than there are axes in conventional Euclidean space. We must find suitable criteria for fixing these azimuthal vector fields in numerical simulations, so that they reduce to the standard rotation generators when considered on a metric sphere.

Because angular momentum is generally thought of as a conserved charge associated with rotation symmetry-and indeed the quantity given in (A1) can be shown to be conserved under time evolution [70,72] when $\vec{\phi}$ is a Killing vector of the dynamical horizon world tube-it makes sense to consider Killing's equation to be the essential feature of the azimuthal vector field. If a Killing vector on a dynamical horizon is a tangent to each (twodimensional) apparent horizon, then the vector field must be a Killing vector of each apparent horizon. However in a general spacetime, on an arbitrary apparent horizon, there is no reason to expect any Killing vectors to exist. So in the 
cases of most interest to numerical relativity, when there are no true rotation symmetries, we must relax the symmetry condition and find those vector fields that come "closest" to generating a symmetry of the apparent horizon. In other words, we seek optimal approximate Killing vectors of the apparent horizon.

In [73], a practical method for computing approximate Killing vectors was introduced, which has since been applied on numerous occasions, e.g., [18,19,29,74]. This method involves integrating the Killing transport equations along a predetermined network of coordinate paths. The resulting vector field is guaranteed to be a Killing vector field if such a field exists and coincides with the computed field at any point on the network. However if no true Killing field exists, the integral of the Killing transport equations becomes path-dependent. This means that the computed vector field will depend in an essential way on the network of paths chosen for the integral. Perhaps even more serious, if there is no true Killing field, then the transport of a vector around a closed path will not necessarily be an identity map. As a result, the computed vector field cannot be expected to reduce to any smooth vector field in the limit that the network becomes more refined. This kind of approximate-Killing-vector field is simply not mathematically well-defined in the continuum limit.

Here we will describe a kind of approximate-Killingvector field that, as well as having a well-defined continuum limit, is actually easier to construct than those of the Killing transport method, at least in our particular code. Our method is extremely similar to that described by Cook and Whiting [41] but was actually developed independently by one of the current authors [75].

\section{Zero expansion, minimal shear}

Killing's equation

$$
D_{(A} \phi_{B)}=0
$$

has two independent parts: the condition that $\vec{\phi}$ be expansion-free

$$
\Theta:=\stackrel{\circ}{g} A B D_{A} \phi_{B}=0
$$

and the condition that it be shear-free

$$
\sigma_{A B}:=D_{(A} \phi_{B)}-\frac{1}{2} g_{A B} \Theta=0,
$$

where uppercase Latin letters index the tangent bundle to the two-dimensional surface, $\stackrel{\circ}{g}_{A B}$ is the metric on that surface, and $D_{A}$ is the torsion-free covariant derivative compatible with that metric.

When constructing approximate Killing vectors, a question arises: Which condition is more important, zero expansion or zero shear? Shear-free vector fields (conformal Killing vectors) are simply coordinate rotation generators in the common case of coordinate spheres in a conformally flat space. They are therefore readily available in that context. A very interesting and systematic approach to their use has been given by Korzynski [76], and they have been used in the construction of conformally flat binary-blackhole initial-data sets [28,29]. However, in the case of a general surface in a general spatial slice, the conformal Killing vectors are not known a priori, and they are more difficult to construct than expansion-free vector fields. Expansion-free vector fields have the additional benefit of providing a gauge-invariant spin measure on a dynamical horizon [70], ${ }^{10}$ so we restrict attention to the expansionfree case.

Any smooth, expansion-free vector field tangent to a topological two-sphere can be written as

$$
\phi^{A}=\epsilon^{A B} D_{B} z,
$$

where $\epsilon^{A B}$ is the Levi-Civita tensor and $z$ is some smooth potential function.

We assume that the function $z$ has one local maximum, one local minimum, and no other critical points. This is equivalent to the assumption that the orbits of $\vec{\phi}$ are simple closed loops. In order for $\phi^{A} \phi_{A}$ to have the proper dimensions, $z$ must have dimensions of area. For the case of the standard rotation generators of the metric two-sphere, the three $z$ functions are the three $\ell=1$ spherical harmonics, multiplied by the square of the areal radius of the sphere.

Within this space of expansion-free vector fields, we would now like to minimize the following positive-definite norm of the shear:

$$
\|\sigma\|^{2}:=\oint_{\mathcal{H}} \sigma_{B C} \sigma^{B C} d A
$$

Substituting Eq. (A5) for $\vec{\phi}$ in this expression and integrating twice by parts, $\|\sigma\|^{2}$ takes the form of an expectation value:

$$
\|\sigma\|^{2}=\oint_{\mathcal{H}} z H z d A,
$$

where $H$ is the self-adjoint fourth-order differential operator defined by

$$
H z=D^{4} z+\stackrel{\circ}{R} D^{2} z+D^{A} \stackrel{\circ}{R} D_{A} z,
$$

and $D^{2}$ is the Laplacian on the (not necessarily round) sphere, $D^{4}$ is its square, and $\stackrel{\circ}{R}$ is the Ricci scalar curvature of the sphere. In our sign convention, $\stackrel{\circ}{R}=2$ on the unit sphere, so we can immediately see that $\mathrm{Hz}=0$ when $z$ is

\footnotetext{
${ }^{10}$ The dynamical horizon is essentially the world tube foliated by the apparent horizons. The gauge freedom is that of extending the foliation off of this world tube. The fact that this gauge invariance occurs when $\vec{\phi}$ is expansion-free can most easily be shown by expressing the factor $s^{j} K_{i j}$ in Eq. (A1) in terms of the ingoing and outgoing null normals to the two-surface. The boost freedom in these null normals has no effect on the spin when $\vec{\phi}$ is expansion-free.
} 
an $\ell=1$ spherical harmonic and therefore that their associated vector fields are shear-free.

It is now tempting to minimize the functional $\|\sigma\|^{2}$ in (A7) with respect to $z$. However, doing so will simply return the condition that $z$ lie in the kernel of $H$. If there are no true Killing vectors, this will mean that $z$ is a constant and therefore that $\vec{\phi}$ vanishes. We need to restrict the minimization procedure to cases that satisfy some normalization condition. In this case, we require that the norm of the vector field

$$
\oint_{\mathcal{H}} \phi^{A} \phi_{A} d A
$$

take some given positive value. This restriction can be made with the use of a Lagrange multiplier. Specifically, the functional we wish to minimize is

$$
I[z]:=\oint_{\mathcal{H}} z H z d A+\lambda\left(\oint_{\mathcal{H}} D^{A} z D_{A} z d A-N\right)
$$

for some yet undetermined positive parameter $N$. Note that $\lambda$ is the Lagrange multiplier and we have made use of the fact that Eq. (A5) implies that $\vec{\phi} \cdot \vec{\phi}=\vec{D} z \cdot \vec{D} z$. Minimizing the functional $I$ with respect to $z$ returns a generalized eigenvalue problem:

$$
H z=\lambda D^{2} z
$$

It is at this point that we can most easily clarify the difference between our construction of approximate Killing vectors and that of Cook and Whiting in [41]. The difference lies in the choice of norm in which the minimization problem is restricted. Rather than fixing the norm (A9) to take some fixed value in the minimization, Cook and Whiting instead fix the dimensionless norm:

$$
\oint_{\mathcal{H}} \stackrel{\circ}{R} \phi^{A} \phi_{A} d A .
$$

In general, we see no particular reason to prefer either norm over the other, but for the current purposes we have at least an aesthetic preference for (A9), which is positivedefinite even at high spin, whereas (A12) is not, because the scalar curvature $\stackrel{\circ}{R}$ of the horizon becomes negative near the poles at high spin. If the norm (A9) in Eq. (A10) is replaced by (A12), the result is the problem described in [41]:

$$
H z=\lambda\left(\stackrel{\circ}{R} D^{2} z+D^{A} \stackrel{\circ}{R} D_{A} z\right) .
$$

In our numerical code, we discretize (A11) [or, optionally, (A13), but not for any results published here] and solve the resulting linear algebra problem with a LAPACK routine [77]. Note, however, one technical peculiarity: The operators $H$ and $D^{2}$ in (A11) share a kernel, the space of constant functions. This means that this generalized eigen- value problem is singular, a fact that can cause considerable difficulties for the numerical solution [78]. The same can be said of (A13). For our purposes, this complication is easily evaded. Since we are working with a spectral code, it is easiest to discretize the problem using expansion into the spectral basis functions (coordinate spherical harmonics). When this is done, the space of constant functions-the shared kernel of the two operators - is simply the span of a single basis function: the constant $Y_{00}$. This basis function can easily be left out of the spectral expansion and thereby removed from the numerical problem.

Expansion into coordinate spherical harmonics has another practical advantage. As noted earlier, for metric spheres in standard coordinates, the Killing vectors arise when $z$ is given by an $\ell=1$ spherical harmonic. Thus, assuming our horizon is nearly round, and noticeably so in the given coordinates, the lowest basis functions (the $\ell=1$ spherical harmonics) should nicely approximate the intended eigenfunctions. The higher basis functions should simply provide small corrections.

In summary, the approach that we take to finding approximate Killing vectors begins with a spectral decomposition of Eq. (A11). This problem, of course, provides as many eigenvectors as there are elements of the spectral decomposition. We restrict attention to the three eigenvectors with smallest eigenvalues (ignoring the vector corresponding to the constant eigenfunction, which is physically irrelevant and removed from discretization), as these are the ones corresponding to vector fields with the smallest shear, and, at least for spheres that are only slightly deformed, the orbits of these vector fields are smooth closed loops.

It must be noted that only the eigenvector with the smallest eigenvalue corresponds to a vector field with strictly minimum shear: Even locally, all other eigenvectors are saddle points of the minimization problem. The three of them taken together, however, provide a geometrically defined subspace of the vector space of expansionfree vector fields, a natural generalization of the rotation generators on metric spheres. Using these three vector fields (normalized as described in the next subsection), one can define "components" of the spin angular momentum of a black hole ${ }^{11}$ and from these components infer the spin around an arbitrary axis or even a spin "magnitude" using a metric on this three-dimensional space of generalized rotation generators. In practice, we have found no need to go quite so far. As mentioned in [41], the approximate Killing vectors generally adapt themselves so well to the horizon that one of the components is much larger than the other two, so this is considered the spin magnitude, and

\footnotetext{
${ }^{11}$ In fact, using the higher eigenvectors, one could in principle compute higher-order multipole moments. We see this as a natural extension of the method laid out in [74] for defining the higher multipole moments of axisymmetric black holes.
} 
the associated approximate Killing vector is considered to define the spin axis.

\section{Normalization}

Solutions to the eigenproblem (A11) can only determine the approximate Killing vectors up to a constant scaling. Fixing this scaling is equivalent to fixing the value of $N$ in (A10). The standard rotation generators of metric spheres are normalized such that, when considered as differential operators along their various orbits, they differentiate with respect to a parameter that changes by a value of $2 \pi$ around each orbit. Naively one would like to fix the normalization of approximate Killing vectors in the same way, but a subtlety arises: We can only rescale the vector field by a fixed, constant value. Rescaling differently along different orbits would introduce extraneous shear and would remove the vector field from the pure eigenspace of (A11) in which it initially resided. If an approximate-Killing-vector field has different parameter circumferences around different orbits, then it is impossible to rescale it such that the parameter distance is $2 \pi$ around every orbit. The best one can ask is that $2 \pi$ is the average of the distances around the various orbits.

To consider this in detail, introduce a coordinate system, topologically the same as the standard spherical coordinates on the metric sphere but adapted to the potential function $z$ so that the latitude lines are the level surfaces of $z$ (and, in particular, the poles are at the two critical points we have assumed $z$ to have). More precisely, choose $z$ for the zenith coordinate on the sphere and an arbitrary rotational coordinate-say, the azimuthal angle in the encompassing spatial slice, describing rotations about the axis connecting the critical points of $z$-for the azimuthal coordinate $\varphi$ on the sphere. If the parameter $\tau$ is defined such that $\vec{\phi}=(d / d \tau)_{z=\text { const }}$, then in the basis related to these coordinates, the components of $\vec{\phi}$ are

$$
\begin{gathered}
\phi^{z}(z, \varphi)=\left(\frac{d z}{d \tau}\right)_{z=\mathrm{const}}=0, \\
\phi^{\varphi}(z, \varphi)=\left(\frac{d \varphi}{d \tau}\right)_{z=\mathrm{const}} .
\end{gathered}
$$

Around a closed orbit $\mathcal{C}(z)$, at fixed $z$, the parameter $\tau$ changes by a value of

$$
\begin{aligned}
\tau(z) & =\int_{\mathcal{C}(z)} \frac{d \varphi}{\phi^{\varphi}(z, \varphi)} \\
& =\int_{\mathcal{C}(z)} \frac{d \varphi}{\epsilon^{\varphi z} \partial_{z} z} \\
& =\int_{\mathcal{C}(z)} \sqrt{\stackrel{\circ}{g}} d \varphi,
\end{aligned}
$$

where $\stackrel{\circ}{g}$ is the determinant of the surface metric, evaluated in the $(z, \varphi)$ coordinates. Note that Eq. (A18) follows from Eq. (A17) by the fact that the condition $\stackrel{\circ}{g}_{A B} \stackrel{\circ}{g}_{C D} \epsilon^{A C} \epsilon^{B D}=$ 2 implies $\epsilon^{\varphi z}=1 / \sqrt{\mathrm{g}}$. The average value of $\tau$, over the various orbits, is

$$
\begin{aligned}
\langle\tau\rangle & =\frac{1}{z_{\max }-z_{\min }} \int_{z_{\min }}^{z_{\max }} \int_{\mathcal{C}(z)} \sqrt{\mathrm{o}} d \varphi d z \\
& =\frac{A}{z_{\max }-z_{\min }},
\end{aligned}
$$

where $A$ is the surface area of the apparent horizon. Requiring this average to equal $2 \pi$, we arrive at the normalization condition:

$$
2 \pi\left(z_{\max }-z_{\min }\right)=A .
$$

This normalization condition requires finding the minimum and maximum values of the function $z$, which is only computed on a discrete grid. In our spectral code, in particular, this numerical grid is quite coarse, so numerical interpolation is needed, in combination with an optimization routine. We have implemented such routines to search for $z_{\min }$ and $z_{\max }$, but a numerically cheaper normalization condition would be of interest. Such a condition arises when one assumes that the black hole under consideration is approximately Kerr. In the Kerr metric, for the function $z$ generating the true rotation generator of the Kerr horizon, the following identity holds:

$$
\oint_{\mathcal{H}}\left(z-\langle\langle z\rangle)^{2} d A=\frac{A^{3}}{48 \pi^{2}},\right.
$$

where $\langle\langle z\rangle$ is the average of $z$ over the sphere. The existence of an identity of this form is somewhat nontrivial: The fact that the right side is given purely by the horizon area, and that it does not involve the spin of the Kerr hole, is what makes this identity useful as a normalization condition. This normalization is much easier to impose and requires significantly less numerical effort.

To close the discussion of spin computed from approximate Killing vectors, we demonstrate the effectiveness of the method in a simple test case: an analytic Kerr black hole in slightly deformed coordinates. We begin with a Kerr black hole of dimensionless spin parameter $\chi=1 / 2$, in Kerr-Schild coordinates, but we rescale the $x$ axis by a factor of 1.1. This rescaling of the $x$ coordinate causes the coordinate rotation vector $x \partial_{y}-y \partial_{x}$ to no longer be the true, geometrical rotation generator. Indeed, when we compute the quasilocal angular momentum (A1) on the horizon using this coordinate vector, the result converges to a physically inaccurate value, as demonstrated by the black dotted curve in Fig. 20. If, however, the approximate Killing vectors described above are used, the result is not only convergent but physically accurate. Because the accuracy is slightly better with the normalization condition of 


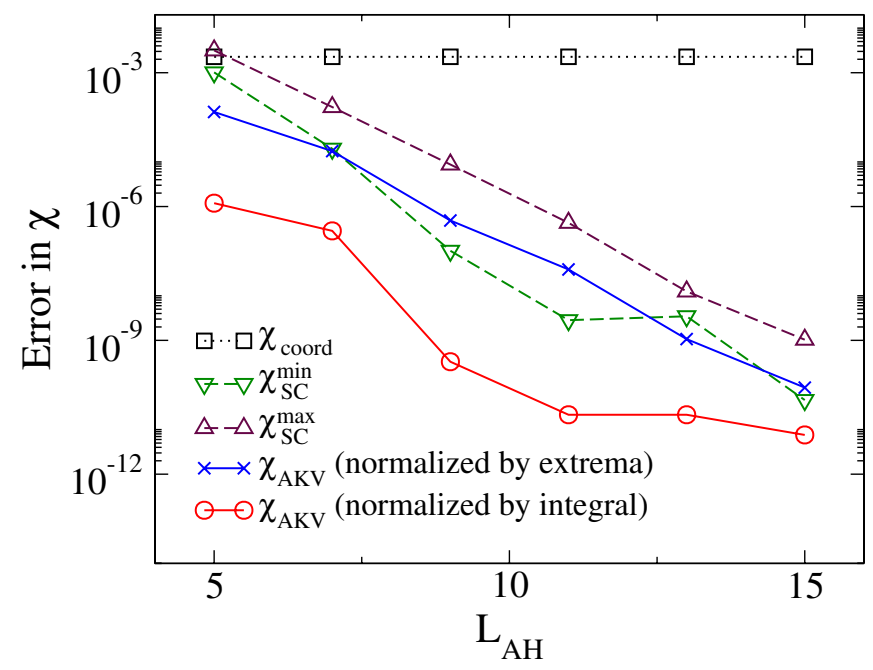

FIG. 20 (color online). Error, relative to the analytic solution, of the spin on the horizon of a Kerr black hole in slightly deformed coordinates. The vertical axis represents $\mid \chi_{\text {computed }}-$ $\chi_{\text {analytic }} \mid$, and data are shown for the spin computed with the standard coordinate rotation vector (in deformed coordinates, so not a true Killing vector) and with our AKVs using both the extremum norm Eq. (A21) and the integral norm Eq. (A22). The spin computed from the coordinate rotation vector quickly converges to a physically inaccurate result. The spin from approximate Killing vectors converges in resolution $L_{\mathrm{AH}}$ to the correct value $\chi=1 / 2$. Curves are also shown for the two spin measures defined in Appendix B. These spin measures also converge exponentially to the physically correct result.

Eq. (A22), that is the condition we use for all results presented in this paper.

\section{APPENDIX B: SCALAR-CURVATURE SPIN}

In this appendix, we define a spin measure in terms of the intrinsic geometry of the horizon, which we compare with the AKV spin in Sec. V. The AKV spin described in Appendix A is a well-defined measure of black-hole spin, even when the holes' horizons have only approximate symmetries. At times sufficiently before or after the holes merge, however, the horizons will not be too tidally distorted and thus will not be too different from the exactly axisymmetric horizons of Kerr black holes.

By assuming that the geometric properties of the horizon behave precisely as they do for a Kerr black hole, one can infer the hole's spin from those properties. For instance, it is common to measure polar and equatorial circumferences of the apparent horizon; the spin is then obtained by finding the Kerr spacetime with the same circumferences [79-81].

To avoid introducing coordinate dependence by defining "polar" and "equatorial" planes, we infer the spin from the horizon's intrinsic scalar curvature $\stackrel{\circ}{R}$. The horizon scalar curvature $\stackrel{\circ}{R}$ has previously been studied analytically for Kerr-Newman black holes [82] and for Kerr black holes perturbed by a distant moon [83]. Numerical studies of $\stackrel{\circ}{R}$ have focused attention on the quasinormal ringing of single, perturbed, black holes [79] as well as on the shape of the individual and common event horizons in Misner data [84]. To our knowledge, the scalar curvature $\stackrel{\circ}{R}$ has not been previously used to infer the horizon spin in numerical simulations.

At a given point on a Kerr black hole's horizon, the horizon scalar curvature $\stackrel{\circ}{R}$ depends only on the hole's mass $M$ and spin $S$. The extrema of $\stackrel{\circ}{R}$ can be expressed in terms of the irreducible mass and dimensionless spin of the Kerr black hole via Eqs. (1) and (2) as

$$
\begin{aligned}
\min (\stackrel{\circ}{R}) & =\frac{-1+2 \sqrt{1-\chi^{2}}}{2 M_{\mathrm{irr}}^{2}}, \\
\max (\stackrel{\circ}{R}) & =-\frac{2}{M_{\mathrm{irr}}^{2} \chi^{4}}\left(-2+\chi^{2}+2 \sqrt{1-\chi^{2}}\right) .
\end{aligned}
$$

Solving for $\chi$ and requiring it to be real yields $\chi$ as a function of $M_{\text {irr }}$ and either $\min (\stackrel{\circ}{R})$ or $\max (\stackrel{\circ}{R})$. We take these functions as definitions of the spin, even when the spacetime is not precisely Kerr:

$$
\begin{aligned}
\left(\chi_{\mathrm{SC}}^{\min }\right)^{2} & :=1-\left[\frac{1}{2}+M_{\mathrm{irr}}^{2} \min (\stackrel{\circ}{R})\right]^{2}, \\
\left(\chi_{\mathrm{SC}}^{\max }\right)^{2} & :=\frac{-2+2 \sqrt{2 M_{\mathrm{irr}}^{2} \max (\stackrel{\circ}{R})}}{M_{\mathrm{irr}}^{2} \max (\stackrel{\circ}{R})} .
\end{aligned}
$$

The definitions of the spin given by Eqs. (B2a) and (B2b) are manifestly independent of spatial coordinates and are well-defined for black holes that are tidally deformed. Also, as they only involve the intrinsic two-dimensional geometry of the apparent horizon, they are also manifestly independent of boost gauge, in the sense described in the previous appendix.

We expect $\chi_{\mathrm{SC}}^{\min }$ and $\chi_{\mathrm{SC}}^{\max }$ to be reasonable measures only if tidal forces can be neglected. Tidal forces scale with the cube of the separation of the holes; for binary with holes of equal mass $M$ and separation $d$, tidal coupling is negligible when $\max (\stackrel{\circ}{R})-\min (\stackrel{\circ}{R}) \gg M / d^{3}$.

We find it convenient to compute $\stackrel{\circ}{R}$ from (i) the scalar curvature $R$ associated with the three-dimensional metric $g_{i j}$ of the spatial slice $\Sigma$ and (ii) the outward-pointing unitvector field $s^{i}$ that is normal to $\mathcal{H}$. This can by done by means of Gauss's equation [e.g., Eq. (D.51) of Ref. [85] (note that the Riemann tensor in Ref. [85] disagrees with ours by an overall sign)]

$$
\stackrel{\circ}{R}=R-2 R_{i j} s^{i} s^{j}-\stackrel{\circ}{K}^{2}+\stackrel{\circ}{K}^{i j} \stackrel{\circ}{K}_{i j},
$$

where $R_{i j}$ and $R$ were defined after Eq. (11) and where $\stackrel{\circ}{K}_{i j}$ denotes the extrinsic curvature of the apparent horizon $\mathcal{H}$ 
embedded in $\Sigma$ (not to be confused with $K_{i j}$, the extrinsic curvature of the slice $\Sigma$ embedded in $\mathcal{M}$ ). The horizon extrinsic curvature is given by

$$
\stackrel{\circ}{K}_{i j}=\nabla_{i} s_{j}-s_{i} s^{k} \nabla_{k} s_{j} .
$$

Inserting Eq. (B4) into Eq. (B3) shows that $\stackrel{\circ}{R}$ can be evaluated exclusively in terms of quantities defined on the three-dimensional spatial slice $\Sigma$.
The accuracy of these spin measures is demonstrated in Fig. 20, which shows a Kerr black hole with $\chi=1 / 2$ in slightly deformed coordinates so that the coordinate rotation vector no longer generates a symmetry. Again, both $\chi_{\mathrm{SC}}^{\min }$ and $\chi_{\mathrm{SC}}^{\max }$ converge exponentially to the physically accurate result.
[1] M. Volonteri, P. Madau, E. Quataert, and M. J. Rees, Astrophys. J. 620, 69 (2005).

[2] C.F. Gammie, S.L. Shapiro, and J.C. McKinney, Astrophys. J. 602, 312 (2004).

[3] S. L. Shapiro, Astrophys. J. 620, 59 (2005).

[4] J.-M. Wang, Y.-M. Chen, L.C. Ho, and R. J. McLure, Astrophys. J. 642, L111 (2006).

[5] J. E. McClintock, R. Shafee, R. Narayan, R. A. Remillard, S. W. Davis, and L.-X. Li, Astrophys. J. 652, 518 (2006).

[6] A. R. King and J.E. Pringle, Mon. Not. R. Astron. Soc. 373, L90 (2006).

[7] A. R. King, J. E. Pringle, and J. A. Hofmann, Mon. Not. R. Astron. Soc. 385, 1621 (2008).

[8] E. Berti and M. Volonteri, arXiv:0802.0025v2.

[9] M. Koppitz, D. Pollney, C. Reisswig, L. Rezzolla, J. Thornburg, P. Diener, and E. Schnetter, Phys. Rev. Lett. 99, 041102 (2007).

[10] M. Campanelli, C. O. Lousto, Y. Zlochower, and D. Merritt, Phys. Rev. Lett. 98, 231102 (2007).

[11] J. A. Gonzalez, M.D. Hannam, U. Sperhake, B. Brügmann, and S. Husa, Phys. Rev. Lett. 98, 231101 (2007).

[12] F. Herrmann, I. Hinder, D. Shoemaker, P. Laguna, and R. A. Matzner, Astrophys. J. 661, 430 (2007).

[13] D.-I. Choi, B. J. Kelly, W. D. Boggs, J. G. Baker, J. Centrella, and J. van Meter, Phys. Rev. D 76, 104026 (2007).

[14] M. Campanelli, C. O. Lousto, Y. Zlochower, and D. Merritt, Astrophys. J. Lett. 659, L5 (2007).

[15] B. Brügmann, J. A. González, M. Hannam, S. Husa, and U. Sperhake, Phys. Rev. D 77, 124047 (2008).

[16] J. G. Baker, W.D. Boggs, J. Centrella, B. J. Kelly, S. T. McWilliams, M. C. Miller, and J. R. van Meter, Astrophys. J. 668, 1140 (2007).

[17] J.D. Schnittman, A. Buonanno, J.R. van Meter, J. G. Baker, W. D. Boggs, J. Centrella, B.J. Kelly, and S. T. McWilliams, Phys. Rev. D 77, 044031 (2008).

[18] M. Campanelli, C. O. Lousto, Y. Zlochower, B. Krishnan, and D. Merritt, Phys. Rev. D 75, 064030 (2007).

[19] M. Campanelli, C. O. Lousto, and Y. Zlochower, Phys. Rev. D 74, 084023 (2006).

[20] M. Campanelli, C. O. Lousto, and Y. Zlochower, Phys. Rev. D 74, 041501(R) (2006).

[21] F. Herrmann, I. Hinder, D. M. Shoemaker, P. Laguna, and R. A. Matzner, Phys. Rev. D 76, 084032 (2007).
[22] P. Marronetti, W. Tichy, B. Brügmann, J. González, and U. Sperhake, Phys. Rev. D 77, 064010 (2008).

[23] E. Berti, V. Cardoso, J. A. Gonzalez, U. Sperhake, and B. Brügmann, Classical Quantum Gravity 25, 114035 (2008).

[24] S. Brandt and B. Brügmann, Phys. Rev. Lett. 78, 3606 (1997).

[25] S. Dain, C. O. Lousto, and Y. Zlochower, Phys. Rev. D 78, 024039 (2008).

[26] S. Dain, C. O. Lousto, and R. Takahashi, Phys. Rev. D 65, 104038 (2002).

[27] G. B. Cook, Phys. Rev. D 65, 084003 (2002).

[28] G. B. Cook and H. P. Pfeiffer, Phys. Rev. D 70, 104016 (2004).

[29] M. Caudill, G. B. Cook, J. D. Grigsby, and H. P. Pfeiffer, Phys. Rev. D 74, 064011 (2006).

[30] E. Gourgoulhon, P. Grandclément, and S. Bonazzola, Phys. Rev. D 65, 044020 (2002).

[31] P. Grandclément, E. Gourgoulhon, and S. Bonazzola, Phys. Rev. D 65, 044021 (2002).

[32] J. W. York, Phys. Rev. Lett. 82, 1350 (1999).

[33] H. P. Pfeiffer and J. W. York, Phys. Rev. D 67, 044022 (2003).

[34] H. P. Pfeiffer and J.W. York, Jr., Phys. Rev. Lett. 95, 091101 (2005).

[35] T.W. Baumgarte, N. O'Murchadha, and H.P. Pfeiffer, Phys. Rev. D 75, 044009 (2007).

[36] D. M. Walsh, Classical Quantum Gravity 24, 1911 (2007).

[37] R. A. Matzner, M. F. Huq, and D. Shoemaker, Phys. Rev. D 59, 024015 (1998).

[38] P. Marronetti and R. A. Matzner, Phys. Rev. Lett. 85, 5500 (2000).

[39] G. Lovelace, Ph.D. thesis, California Institute of Technology, 2007, http://etd.caltech.edu/etd/available/ etd-05232007-115433.

[40] K. Taniguchi, T. W. Buamgarte, J.A. Faber, and S. L. Shapiro, Phys. Rev. D 74, 041502(R) (2006).

[41] G. B. Cook and B. F. Whiting, Phys. Rev. D 76, 041501(R) (2007).

[42] I. Booth and S. Fairhurst, Phys. Rev. D 77, 084005 (2008).

[43] J. M. Bowen, Gen. Relativ. Gravit. 11, 227 (1979).

[44] J. M. Bowen and J. W. York, Jr., Phys. Rev. D 21, 2047 (1980).

[45] R. Arnowitt, S. Deser, and C. W. Misner, in Gravitation: An Introduction to Current Research, edited by L. Witten (Wiley, New York, 1962), pp. 227-265. 
[46] A. Ashtekar, J. Engle, and D. Sloan, Classical Quantum Gravity 25, 095020 (2008).

[47] G. Cook, Living Rev. Relativity 3, 5 (2000), http://www. livingreviews.org/lrr-2000-5.

[48] J. W. York, Jr. and T. Piran, in Spacetime and Geometry, edited by R. A. Matzner and L. C. Shepley (University of Texas, Austin, 1982), pp. 147-176.

[49] H. P. Pfeiffer, D. A. Brown, L. E. Kidder, L. Lindblom, G. Lovelace, and M. A. Scheel, Classical Quantum Gravity 24, S59 (2007).

[50] A. Garat and R.H. Price, Phys. Rev. D 61, 124011 (2000).

[51] J. A. Valiente Kroon, Phys. Rev. Lett. 92, 041101 (2004).

[52] J. W. York, Jr., in Essays in General Relativity, edited by F. J. Tipler (Academic, New York, 1980), pp. 39-58.

[53] H. P. Pfeiffer, G. B. Cook, and S. A. Teukolsky, Phys. Rev. D 66, 024047 (2002).

[54] W. Tichy, B. Brügmann, M. Campanelli, and P. Diener, Phys. Rev. D 67, 064008 (2003).

[55] S. Nissanke, Phys. Rev. D 73, 124002 (2006).

[56] N. Yunes, W. Tichy, B. J. Owen, and B. Brügmann, Phys. Rev. D 74, 104011 (2006).

[57] N. Yunes and W. Tichy, Phys. Rev. D 74, 064013 (2006).

[58] M. Hannam, S. Husa, B. Brügmann, J. González, and U. Sperhake, Classical Quantum Gravity 24, S15 (2007).

[59] B. J. Kelly, W. Tichy, M. Campanelli, and B. F. Whiting, Phys. Rev. D 76, 024008 (2007).

[60] E. Bonning, P. Marronetti, D. Neilsen, and R. Matzner, Phys. Rev. D 68, 044019 (2003).

[61] G. B. Cook and J. W. York, Jr., Phys. Rev. D 41, 1077 (1990).

[62] H. P. Pfeiffer, L.E. Kidder, M.A. Scheel, and S. A. Teukolsky, Comput. Phys. Commun. 152, 253 (2003).

[63] H. P. Pfeiffer, Ph.D. thesis, Cornell University, 2003.

[64] M. Hannam, S. Husa, B. Brügmann, J. A. Gonzalez, and U. Sperhake, Classical Quantum Gravity 24, S15 (2007).

[65] M. A. Scheel, H. P. Pfeiffer, L. Lindblom, L. E. Kidder, O. Rinne, and S. A. Teukolsky, Phys. Rev. D 74, 104006 (2006).
[66] M. Boyle, D. A. Brown, L. E. Kidder, A. H. Mroué, H. P. Pfeiffer, M. A. Scheel, G. B. Cook, and S. A. Teukolsky, Phys. Rev. D 76, 124038 (2007).

[67] L.E. Kidder, Phys. Rev. D 52, 821 (1995).

[68] L. Lindblom, M. A. Scheel, L.E. Kidder, R. Owen, and O. Rinne, Classical Quantum Gravity 23, S447 (2006).

[69] F. Foucart, L.E. Kidder, H. P. Pfeiffer, and S. A. Teukolsky, Phys. Rev. D 77, 124051 (2008).

[70] J.D. Brown and J.W. York, Phys. Rev. D 47, 1407 (1993).

[71] A. Ashtekar, C. Beetle, and J. Lewandowski, Phys. Rev. D 64, 044016 (2001).

[72] A. Ashtekar and B. Krishnan, Phys. Rev. D 68, 104030 (2003).

[73] O. Dreyer, B. Krishnan, D. Shoemaker, and E. Schnetter, Phys. Rev. D 67, 024018 (2003).

[74] E. Schnetter, B. Krishnan, and F. Beyer, Phys. Rev. D 74, 024028 (2006).

[75] R. Owen, Ph.D. thesis, California Institute of Technology, 2007, http://resolver.caltech.edu/CaltechETD:etd05252007-143511.

[76] M. Korzynski, Classical Quantum Gravity 24, 5935 (2007).

[77] http://www.netlib.org/lapack.

[78] E. Anderson, Z. Bai, C. Bischof, S. Blackford, J. Demmel, J. Dongarra, J. DuCroz, A.G. Hammarling, S. Hammarling, A. McKenney, and D. Sorensen, LAPACK Users' Guide (Society for Industrial and Applied Mathematics, Philadelphia, 1999).

[79] P. Anninos, D. Bernstein, S. R. Brandt, D. Hobill, E. Seidel, and L. Smarr, Phys. Rev. D 50, 3801 (1994).

[80] S. R. Brandt and E. Seidel, Phys. Rev. D 52, 870 (1995).

[81] M. Alcubierre et al., Phys. Rev. D 72, 044004 (2005).

[82] L. Smarr, Phys. Rev. D 7, 289 (1973).

[83] J. B. Hartle, Phys. Rev. D 9, 2749 (1974).

[84] J. Massó, E. Seidel, W.-M. Suen, and P. Walker, Phys. Rev. D 59, 064015 (1999).

[85] S. Carroll, Spacetime and Geometry: An Introduction to General Relativity (Addison Wesley, New York, 2003). 